\title{
EVALUATION OF A BIBLIOTHERAPY-BASED STRESS MANAGEMENT TRAINING FOR TEACHERS
}

A Dissertation Presented to
the Faculty of the Graduate School
University of Missouri-Columbia
In Partial Fulfillment
of the Requirements for the Degree
Doctor of Philosophy
by
COLLEEN L. EDDY
Dr. Keith C. Herman, Dissertation Supervisor
MAY 2021


The undersigned, appointed by the dean of the Graduate School, have examined the dissertation entitled

\section{EVALUATION OF A BIBLIOTHERAPY-BASED STRESS MANAGEMENT TRAINING FOR TEACHERS}

presented by Colleen L. Eddy, a candidate for the degree of doctor of philosophy, and hereby certify that, in their opinion, it is worthy of acceptance.

Professor Keith Herman

Professor Wendy Reinke

Professor Francis Huang

Professor Chris Slaten

Professor T. Chris Riley Tillman

Professor James Sebastian 
To all the teachers who have given so much to me throughout my life.

You are amazing. Thank you! 


\section{ACKNOWLEDGEMENTS}

I would like to thank Dr. Herman and Dr. Reinke for creating the Stress Management Training for Teachers book and doing so much to make this project possible. I would also like to thank Dr,'s Huang, Sebastian, Slaten, and Riley-Tillman for all of the feedback to improve the study. This research was funded by an internal research grant from the University of Missouri and supported by the wonderful team at the Missouri Prevention Science Institute. 
TABLE OF CONTENTS

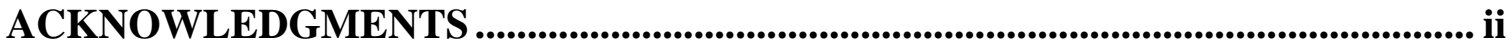

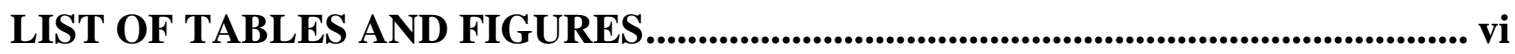

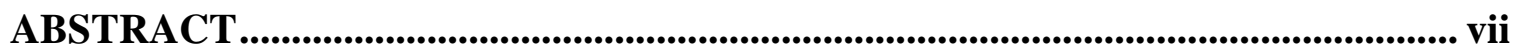

CHAPTER I: INTRODUCTION ...........................................................................1

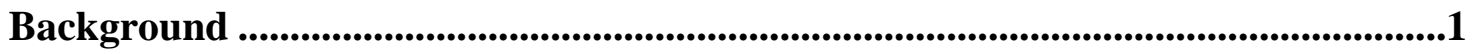

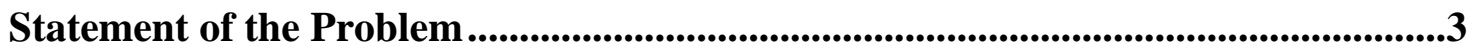

Purpose of the Current Study ....................................................................................6

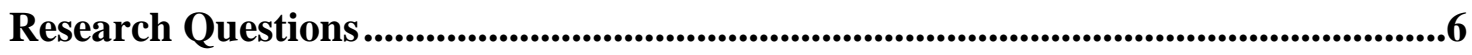

CHAPTER II: LITERATURE REVIEW ..................................................................8

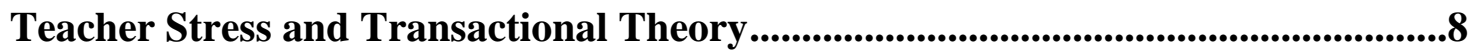

Overview and Prevalence of Teacher Stress .....................................................

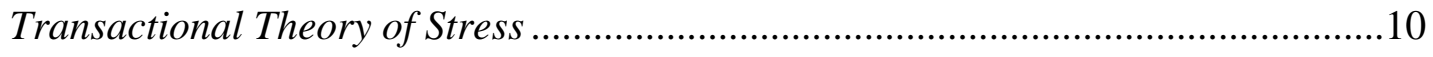

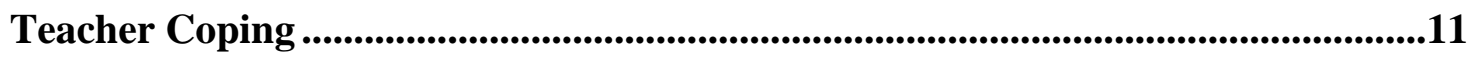

Negative Results of Teacher Stress.................................................................15

Impact of Teacher Stress on Students ............................................................... 15

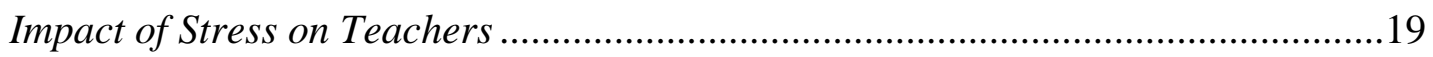

Impact of Teacher Stress on the Educational System ..........................................20

Existing Teacher Stress Reduction Programs ................................................................21

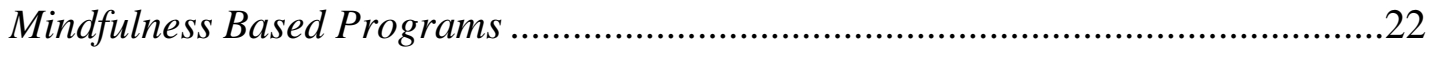

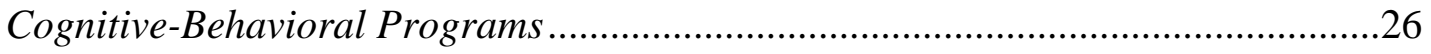

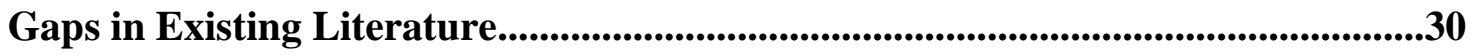

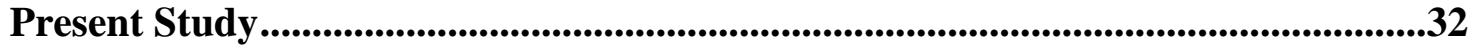




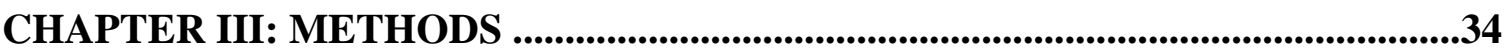

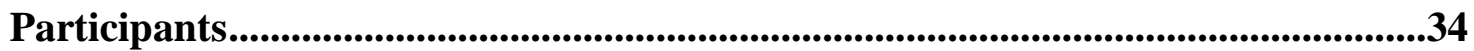

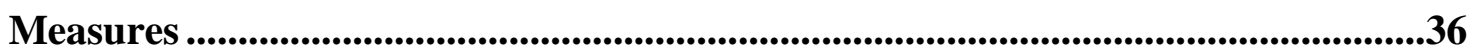

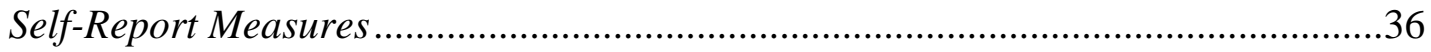

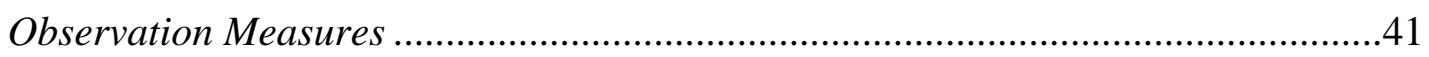

Procedures .................................................................................................................................................42

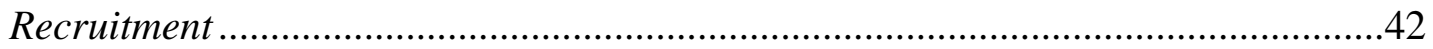

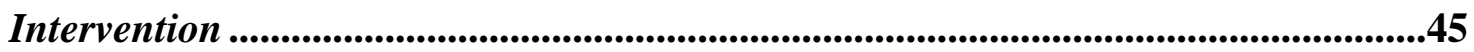

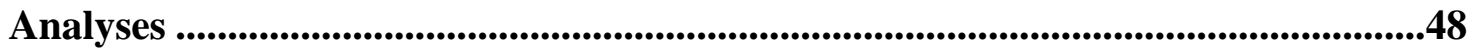

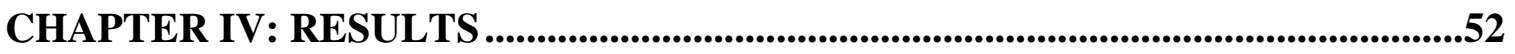

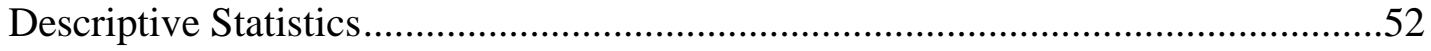

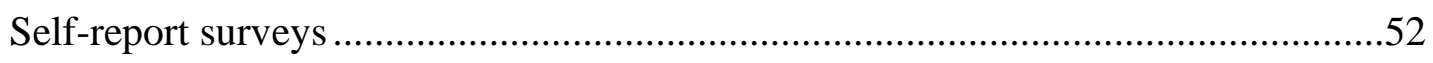

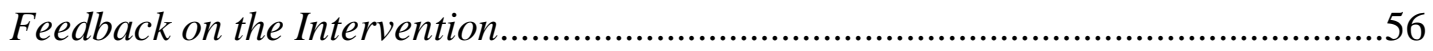

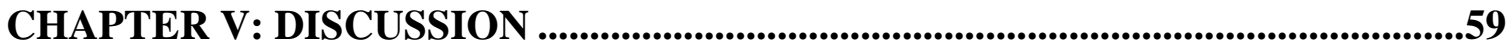

Initial Program Efficacy ............................................................................................................59

Comparison to other Teacher Stress Programs.......................................................65

Limitations...................................................................................................................................67

Future Directions .....................................................................................................................69

Conclusion and Summary ...........................................................................................................72

References.............................................................................................................................74

Appendices..................................................................................................................................90

Appendix A. .....................................................................................................................91

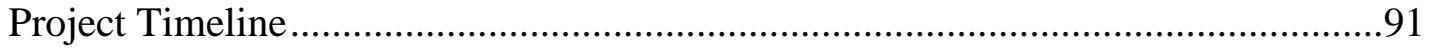




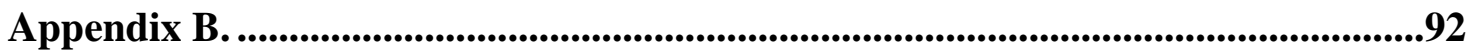

Weekly Self-Monitoring Form ……………………...........................................92

Appendix C............................................................................................................................................93

Theory of Change for Stress Management Intervention...........................................93

Appendix D. .......................................................................................................................................94

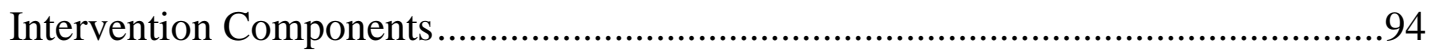

Appendix E........................................................................................................................95

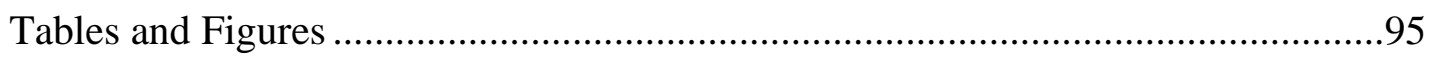

Appendix F. .....................................................................................................................................106

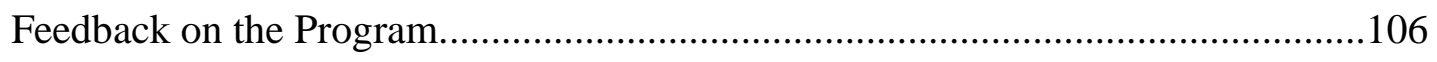

VITA..........................................................................................................................................109 


\section{LIST OF TABLES AND FIGURES}

Table 1 Participant Demographic Information.................................. 93

Table 2 Study Measures................................................... 94

Table 3 Treatment Effects for Stress and Burnout..............................95

Table 4 Treatment Effects for Coping Strategies............................... 95

Table 5 Treatment Effects for Efficacy, Organizational Health, and Job Satisfaction ....96

Table 6 Treatment Effects for Mental Health Screening..........................96

Table 7 Treatment Effects for Observed Teacher Behaviors........................96

Table 8 Growth Modelling of Stress and Coping over Time ........................97

Table 9 Pre and Post Comparisons of Study Measures ........................... 98

Figure 1 Average Self-Reported Stress over Time $\ldots \ldots \ldots \ldots \ldots \ldots \ldots \ldots \ldots \ldots \ldots \ldots . \ldots 9$

Figure 2 Average Self-Reported Coping over Time............................. 99

Figure 3 Average Self-Reported Confidence in Coping over Time..................100

Figure 4 Average Self-Reported Awareness of Coping Strategies over Time ...........100

Figure 5 Average Self-Reported Understanding of Coping Strategies over Time.......101

Figure 6 Average Self-Reported Use of Coping Strategies over Time................101

Figure 7 Participants' Change in Anxiety Severity Levels.........................102

Figure 8 Participants' Change in Depression Severity Levels.......................102

Table 10 Control Group Only Pre-Post Comparisons............................103 


\begin{abstract}
The study was a randomized control trial of a cognitive-behavioral bibliotherapy-based stress management training for teachers. The intervention consisted of the Stress Management for Teachers book, an in-person meeting, and three brief follow-up webinars with weekly practice over the course of 4 weeks. There were 53 participants in the study and teachers were randomly assigned to the intervention or waitlist control conditions. Multilevel regression was used to examine the intervention effects accounting for teachers nested in schools and controlling for school district, years of teaching experience, and the baseline measure. The intervention reduced stress on the Perceived Stress Scale $(\beta=-0.53)$. Teachers also increased in specific coping strategies of planning $(\beta=0.62)$, positive reframing $(\beta=0.66)$, and decreased in self-reported substance use $(\beta$ $=-0.44)$. The intervention did not have statistically significant effects on contextual factors or on observed classroom behaviors. The intervention demonstrated treatment effects on anxiety symptoms on the GAD-7 $(\beta=-0.51)$. Further, in comparison to participants in the control group, teachers in intervention condition had statistically significant changes in symptom severity level in anxiety on the GAD-7 (Cramer's V = $.45)$ and in depression on the PHQ-8 (Cramer's $\mathrm{V}=.42)$. From progress monitoring data, teachers in the intervention condition had greater rates of improvement in increased coping, confidence and use of coping strategies ( $p$ 's $<.05)$. Additional supports may be needed to enhance coping with stressors related to classroom management and organizational health and to optimize the intervention delivery.
\end{abstract}




\section{CHAPTER I: INTRODUCTION}

\section{Background}

Teachers contribute greatly to youth development, as schools are typically the main systems children first interact with outside of their homes (Bronfenbrenner \& Morris, 2006; Rimm-Kaufman \& Pianta, 2000). The task of educating youth is essential to the health and growth of society and is the key responsibility of teachers. Beyond providing academic instruction, educators are also responsible for teaching youth how to interact with others in social settings (Davis, 2003; Pianta, Hamre, \& Stuhlman, 2003). One teacher has the potential to impact each student in the classroom, and across the course of a teacher's career, this influence on youth development compounds with each year in the profession. Positive teaching practices and student-teacher relationships are protective and beneficial for students (Hamre \& Pianta, 2001; Roorda, Koomen, Spilt, \& Oort, 2011). The reverse is also true, such that negative interactions with teachers together with existing risk factors can lead to cascading negative outcomes (Masten et al., 2005), such as lower academic engagement and achievement (Roorda et al., 2011), as well as poorer social and emotional outcomes (Jennings \& Greenberg, 2009). Positive relationships with teachers can be protective for students and buffer against existing risk (Gregory \& Ripski, 2008; Rudasill, Reio, Stipanovic, \& Taylor, 2010).

In addition to playing such an important role in youth development, teachers make up a large percentage of the workforce in the United States. In 2017, there were 3.2 million full-time public school teachers in the United States (National Center for Education Statistics Institute of Education Sciences, 2018). The high populations of 
teachers makes it one of the top five largest occupations in the country, following retail salespersons, which included 4.4 million workers in 2017 and greater than 2.9 million registered nurses (U. S. Bureau of Labor Statistics, 2018). Given the sheer number of teachers in the profession, the needs of teachers are critical to consider from an occupational perspective.

Despite the importance and the large percentage of workers who are teachers, there are many challenges facing the profession today, and this contributes to high levels of stress. The problem of teacher stress has been consistently reported since the 1970s across many countries (Kyriacou, 2001, 2011; Kyriacou \& Sutcliffe, 1978). Teacher stress is a societal concern because it is associated with a wide range of negative outcomes for students, schools, and the broader educational system. Stress and negative emotional factors impact teachers' abilities to provide effective instruction, manage students' behaviors, and model social emotional competencies for students (Arens \& Morin, 2016; Herman, Hickmon-Rosa, \& Reinke, 2018; Jennings \& Greenberg, 2009; McLean \& Connor, 2015). Teacher stress and burnout are associated with negative mental health outcomes including depression, anxiety, and leaving the profession (Shin, Noh, Jang, Park, \& Lee, 2013; Skaalvik \& Skaalvik, 2011). In considering the broader educational system, teacher attrition costs millions of dollars with the need to recruit, rehire, and train new teachers each year (Barnes, Crowe, \& Schaefer, 2007; Ingersoll, 2001; Watlington, Shockley, Guglielmino, \& Felsher, 2010). Though the estimates vary, it is commonly reported that one in five teachers leave the field within the first five years of entering the profession (Ingersoll, 2001), and most often high stress is reported at the reason for leaving the field (Ingersoll \& Smith, 2003). Given the widespread problem of 
teacher stress and associated negative outcomes, evidence-based programs are needed to support teachers to cope with their occupational stress.

\section{Statement of the Problem}

There are a few promising interventions to support teachers to cope with the stress of their jobs, yet these programs have not completed the research and development process necessary to be considered evidence-based interventions (Gottfredson et al., 2015). There have been two main categories of stress management programs for teachers based in psychological theories of cognitive behavioral therapy and mindfulness (Cullen \& Wallace, 2010; Forman, 1982; Jennings et al., 2017). Both types of programs have demonstrated positive effects through initial randomized control efficacy trials, but additional refinement of these programs is needed prior to scale-up and dissemination (Gottfredson et al., 2015). The cognitive behavioral programs were initially developed in the 1980s and were based in stress inoculation theory (Forman, 1982). Initial studies showed that the stress management program for teachers had positive effects on reducing teacher symptoms of stress and mental health symptoms in comparison to control groups as well as in comparison to classroom management training and teacher support groups (Cecil \& Forman, 1990; Forman, 1982; Sharp \& Forman, 1985). Despite these promising results of these early efficacy studies, there has not been additional development of these programs informed by the findings of the studies until recently. Dicke, Elling, Schmeck, and Leutner (2015) found similar effects on stress reduction in comparing a cognitivebehavioral stress management training and classroom management training. These researchers suggest that classroom management training should be included in stress management training for teachers, because it may reduce stress related to student 
behaviors, which is a commonly cited source of teacher stress (Boyle, Borg, Falzon, \& Baglioni, 1995; Dicke et al., 2015).

Despite the efficacy of cognitive-behavioral and classroom management training, most recent development of stress management programs for teachers has shifted to mindfulness based stress management, and have not incorporated classroom management into the development of these programs for teachers (Jennings \& DeMauro, 2017). Initial efficacy studies on two different mindfulness programs for teachers have demonstrated reductions in teacher reported stress and mental health symptoms (Jennings, Frank, Snowberg, Coccia, \& Greenberg, 2013; Roeser et al., 2013). In the largest evaluation study of a mindfulness program, the results showed positive results for teachers who participated in the program as well as improvements in positive observed teacher-student interactions, but with more modest effect sizes in comparison to prior studies (Jennings et al., 2017). These findings suggest possible limitations in existing mindfulness based stress management for teachers. First, the low enrollment could indicate that mindfulness is a specific type of stress management that may work well for some teachers, but may not be desirable for all teachers. Second, the low to moderate effect sizes of the program could also be due to lower effects of relaxation-based stress management programs in comparison to those based in cognitive-behavioral theory (Richardson \& Rothstein, 2008). Overall, mindfulness based stress management is a promising technique for some teachers, but programs using only mindfulness, without additional skills to address sources of stress, may not be sufficient to support all teachers to address varied sources of stress. 
Building on prior research and stress management interventions, Herman and Reinke (2015) created the Stress Management Training for Teachers as a comprehensive model that integrates promising components from existing programs to support teachers to cope with stress. The intervention is delivered through a bibliotherapy, along with one 90 minute in-person training and three online webinars all based on the book content to encourage access and interaction with book resources and activities. Cognitive behavioral theory and social learning theory are the foundational theories for the coping framework for teachers to develop specific skills. The mechanisms for change in the intervention are by increasing the awareness, understanding and use of cognitive and behavioral coping strategies to reduce stress (Herman \& Reinke, 2015). First, teachers develop a better understanding of the function of stress and tools for self-monitoring to develop greater awareness of stress and coping (Harmon, Nelson, \& Hayes, 1980). Then teachers learn strategies to increase adaptive thinking, such as creating coping thoughts, and decreasing negative thoughts, as well as the ABCDE method (Ellis, 1991). The adaptive behavior section includes recommendations related to prior research in stress management training, such as mindfulness strategies (Jennings \& DeMauro, 2017) and increasing competency in classroom management, in order to directly address sources of stress of disruptive student behaviors (Dicke et al., 2015; Sharp \& Forman, 1985). By increasing teachers' with knowledge and awareness of stress, and specific skills to change thoughts and behaviors, this program equips teachers with a wide range of stress management strategies that can be adapted to fit with teachers' personal preferences and unique experiences of stress. 


\section{Purpose of the Current Study}

The purpose of the study is to examine the initial efficacy of the Stress Management Training for Teachers through a randomized control trial. The design of the study will follow the standards for efficacy by the Society of Prevention Research (Gottfredson et al., 2015). The sample included 52 teachers in a Midwestern metropolitan area and surrounding rural areas. The intervention consisted of a bibliotherapy, a therapeutic intervention delivered by reading book, an in-person training, and additional complementary online webinars delivered over a four week period. Teachers were randomly assigned to the treatment or waitlist control condition. The study took place during the academic school year with baseline data collection prior to the intervention and follow-up four to six weeks after the intervention. In order to examine changes over time in response to the intervention, teachers completed weekly ratings of their stress and coping behaviors. The main purpose of the study was to examine the effects of the stress management program on teacher reported stress and coping, long-term outcomes of emotional health and occupational burnout, and contextual factors of teaching efficacy and job satisfaction. In addition to self-report data, classroom-level behavioral observations were completed by trained observers. Overall the purpose of the proposed study is to examine the effects of the Stress Management Training for Teachers under optimal and controlled conditions as an initial efficacy trial, and better understand how changes occur as a result of the intervention.

\section{Research Questions}

1. Does the Stress Management Training for Teachers reduce teacher reported stress and negative mental health outcomes and improve teacher coping and wellbeing? 
2. Does the Stress Management Training for Teachers have an effect on observed teacher-student interactions in the classroom?

3. How does teacher stress and coping change over time as a result of the intervention? 


\section{CHAPTER II: LITERATURE REVIEW}

This section will review relevant research literature about teacher stress. First, key concepts including the transactional theory of stress, burnout, and coping will be defined. Second, theories about the relationship of teacher stress with negative outcomes for the workforce of teachers, as well as the implications for students will be reviewed. Finally, existing stress management training for teachers will be reviewed and areas for further development will be identified to provide the rationale for the existing study of evaluating a novel stress management intervention for teachers.

\section{Teacher Stress and Transactional Theory Overview and Prevalence of Teacher Stress}

Teaching ranks as one of the most stressful professions in comparison with other occupations (Johnson et al., 2005). In samples of teachers across multiple counties, at least $25 \%$ consistently report that teaching is highly or extremely stressful (Kyriacou, 2011). In the United States, a recent study found that $93 \%$ of teachers reported high stress (Herman et al., 2018). Though there may be some differences in individual teachers' experience and the broader educational context may contribute to some variation in the sources of stress, the phenomenon of high stress seems to be widespread in the profession.

Teacher Stress. Early researchers in teacher stress, Kyriacou and Sutcliffe (1978), described the experience as the negative emotions that result from the demanding tasks that are involved in teaching. These demands must be perceived as some sort of threat to the teachers' self-image or self-esteem, a threat to the teachers' or students' physical safety, or an obstacle to accomplishing other tasks (Kyriacou, 2011). Teachers 
may perceive the same situation in different ways, and so there are many potential stressors for teachers (Kyriacou, 2001; Kyriacou \& Sutcliffe, 1978). Sources of stress can be categorized as those that occur within the classroom as a result of working with students and those that occur outside of the classroom from the broader school context and educational system.

Stressors that occur within the classroom when working with students include: managing student behaviors, adapting instruction to meet the diverse needs of all students, motivating students, and having limited time to accomplish necessary teaching tasks (Boyle et al., 1995; Herman \& Reinke, 2015). Teachers are responsible for the academic instruction of students, which can be challenging to accomplish if students are unmotivated or if students have behavioral or emotional needs that can impact their learning and the classroom environment (Jennings \& Greenberg, 2009). When facing immediate stressors in the classroom, teachers are often unable to walk away to calm down or ask for help from another adult, as they are responsible for the supervision and safety of their students (Travers, 2015). For example, when managing a disruptive student's behaviors, a teacher may be in a challenging position of weighing the needs of the individual student against the rest of the students in the classroom. As a result, teachers consistently report managing student behaviors as a major source of stress in the classroom environment (Boyle et al., 1995; Kokkinos, 2007).

In addition to stressors in the classroom, teachers also report sources of stress related to working with other adults in the school system, such as managing challenging colleagues, dealing with parents, and unsupportive administration (Herman \& Reinke, 2015). Further, teachers report stressors related to the broader educational system 
including poor working conditions (i.e., low pay, insufficient resources), changing curricula, and high-stakes assessment (Boyle et al., 1995; Herman \& Reinke, 2015; Kyriacou, 2011). Specifically in the United States, changes to the educational system from the federal mandates of No Child Left Behind (NCLB; 2001), have led to increased use of high-stakes testing and teacher accountability to high test scores, which have contributed to higher test anxiety for teachers and physical manifestations of stress associated with burnout and attrition (Ryan et al., 2017). Dissatisfaction of working conditions such as low pay and stagnant funding for education across the country have recently reached a national tipping point resulting in statewide walkouts in the spring of 2018 in West Virginia, Oklahoma, Kentucky, Colorado, and Arizona with teachers advocating for improvements for working conditions (Turner, Lombardo, \& Logan, 2018). Given the national attention of the problems facing teachers, now is a critical time for effective supports for teachers to cope with the stress of the profession.

\section{Transactional Theory of Stress}

Researchers often conceptualize teacher stress using the model proposed by Kyriacou and Sutcliffe (1978) and based in the transactional theory of stress by Lazarus and Folkman (1984). In this model, stress is defined as a balance between an individual's perceptions of the demands of the environment and his or her perceived coping abilities to meet the demands. According to Lazarus and Folkman (1987), there are three main components and assumptions in this theory. The first main assumption is the importance of the interaction in the relationship between the person and the environment, as emotional experiences require both an environmental stimuli and an individual to respond. The second key concept in the model is the emphasis on the process or the 
changes that occur over time and in different situations. The third key component in this theory is role of cognitive appraisals in mediating the relationship between the environment and the person's response.

According to Lazarus and Folkman (1984), the cognitive appraisal process involves two components. The first is the primary appraisal of the perceived environmental demands, which is the determination if the demand will have an impact on the individual's wellbeing. In order to be perceived as stressful, demands must pose a possible threat, have caused harm, or pose a challenge that could result in potential benefits or gain (Lazarus \& Folkman, 1987). The secondary appraisal is the perception of one's ability to meet the demands in the environment based on the degree of control and the specific strategies or options available to meet the demand. Stress results from a perceived imbalance between environmental demands and coping strategies, when a person believes he or she does not possess adequate coping strategies or resources to meet the environmental demands (Lazarus \& Folkman, 1984, 1987). Though these appraisals are called primary and secondary, there is no distinct temporal order to the appraisal process (Lazarus \& Folkman, 1987), and the appraisal process often occurs many times in a stressful situation.

\section{Teacher Coping}

In the transactional theory, coping strategies are critical to the experience of stress (Lazarus, 2001); coping strategies are important to consider as they are malleable factors that can be supported in order to reduce stress. Coping behaviors moderate the relationship between stressors and resulting burnout, such that infrequent use and use of ineffective coping strategies are associated with higher stress and burnout and in contrast, 
the greater use of effective coping is associated with lower stress and burnout (Montgomery, 2017). Further, in models examining coping and stress together predicting burnout, coping also has a stronger and more consistent statistical association than stress (Eddy, Herman, \& Reinke, 2019). From the transactional theory of stress, coping is a process that occurs over time and in different situations (Lazarus \& Folkman, 1987).

\section{Types of Coping}

Coping is categorized based on two main functions of the coping behaviors to either address the stressor directly, or to manage the experience of stress (Lazarus \& Folkman, 1984). Problem-focused coping has the purpose of changing the environmental stressor by a direct behavioral change or through a change in thinking. Emotion-focused coping is coping in order to reduce or tolerate the physical experience of stress, which may also be through avoidance. Though in most stressful circumstances, individuals engage in both problem and emotion-focused coping, the use of the two types of coping strategies is greatly determined by the appraisals processes; specifically the degree of threat the stressor presents and the perception of control over the stressors (Lazarus \& Folkman, 1987). If a person believes they are capable of addressing the stressor,, if the stakes are particularly high, or if they have some control, they are more likely to engage in problem-focused coping. A person will be more likely to use emotion-focused strategies if he or she perceives the situation as difficult to change(Lazarus, 1980).

Aligning with the transactional theory, Kyriacou (2001) used the terms direct action techniques and palliative techniques to describe teacher coping corresponding with problem-focused and emotion-focused coping, respectively. For teachers, direct-action or problem-focused techniques may include behaviors like reducing students' disruptive 
behaviors or spending additional time preparing for a lesson in order to directly address the source of the stress. Palliative or emotion-focused coping for teachers may be taking deep breaths when engaging in a challenging conversation with a parent or colleague.

More recently, Montgomery and Rupp (2005) used a slightly different characterization for teacher coping based on the type of active or passive strategies to address the stressors (Carver, 1997). According to Montgomery and Rupp (2005), active coping is any action to manage or reduce the stress, and it could be through both problem-focused or emotion-focused strategies. For example, brainstorming possible solutions to handle the situation and taking deep breaths are both active ways to manage the stressor even though problem-solving would be considered a direct action technique and deep breathing would be considered a palliative coping technique (Kyriacou, 2001). Passive coping is defined as avoidance of the stressors such as through wishful thinking, resignation, emotional disengagement, or procrastination (Montgomery, 2017). In a metaanalysis of the relationships among stress, coping and burnout in teachers, Montgomery and Rupp (2005) found that active coping rather than passive coping was associated with better outcomes for teachers, such as preventing professional burnout.

\section{Outcomes of Coping}

Coping can also be described in terms of the impact on the individual and the environment in the short and long term (Lazarus \& Folkman, 1987). In the moment, coping can result in a change in emotional experience, such as feelings of relief, no emotional change, or even increased stress. The environmental stressors are impacted by coping in that the sources of stress may be reduced, they could stay the same, or they could even increase. Coping can also be described as dysfunctional or adaptive based on 
these short-term effects as well as long-term individual outcomes of physical health, job performance, and social functioning (Carver, Scheier, \& Weintraub, 1989; Montgomery, 2017). Different types of coping behaviors are more likely to be associated with positive and negative long-term outcomes (Carver et al., 1989). Specifically for teachers, disengagement and procrastination may cause immediate reductions of stress but over time avoidance is associated with an increased risk for burnout (Montgomery \& Rupp, 2005). Alternatively, social support is associated with more positive long-term outcomes, and therefore may be a more effective method of coping (Griffith, Steptoe, \& Cropley, 1999). Coping strategies that result in positive outcomes for teachers are critical to consider as they can buffer against the negative impacts of stress over time.

\section{Coping-Competence-Context Model of Teacher Stress.}

Recently, Herman, Reinke and Eddy, (2020) proposed a new theory to conceptualize teacher stress and coping called the Coping-Competence-Context Model of Teacher Stress, or the 3C model. This model identifies these three areas as key leverage point for teacher stress and emotional well-being. The first area is individual coping and mindset regarding stress as prior research and theory has demonstrated that how teachers' perceive and manage stress influences teacher outcomes of burnout and self-efficacy (Eddy et al., 2019; Kim, Shin, Tsukayama, \& Park, 2020). The second area influencing teacher stress is classroom practices and competence. This pathway specifically involves teacher-student relationships and classroom management practices, which are critical for creating a positive learning environment for students and also protective for teachers well-being (Bottiani, Duran, Pas, \& Bradshaw, 2019; Corbin, Alamos, Lowenstein, Downer, \& Brown, 2019). The third area is contextual factors that contribute to high 
demands and systemic stressors (e.g., high-stakes testing and insufficient funding for classroom supplies). Each of these three pathways are malleable and conceptualized as essential for improving teacher and student outcomes.

\section{Negative Results of Teacher Stress}

When teachers experience high stress and are not coping well, this can cause problems for the individual teacher (Cropanzano, Rupp, \& Byrne, 2003; Shin et al., 2013) and can impact his or her abilities to create a positive learning environment for students (Herman et al., 2018; Jennings \& Greenberg, 2009). Over time this prolonged stress is associated not only with problems for students in the classroom, and for the individual teacher, but excessive stress also has a negative impact on the educational system (Ingersoll \& Smith, 2003). The following section will review the consequences of stress on three main levels: students, teachers, and on the educational system.

\section{Impact of Teacher Stress on Students}

Teachers have a unique contribution to child development (Davis, 2003), and emotional wellbeing influences teachers' abilities to positively impact students in the classroom (Jennings \& Greenberg, 2009; Oberle \& Schonert-Reichl, 2016). As reviewed by Davis (2003), teachers contribute to the cognitive and intellectual development of children, their motivation and desire to learn, and to their social development and personal identity formation processes. The relationship between teacher wellbeing and student outcomes can be explained through various theories including ecological theory and attachment theory.

Bioecological Model of Development. One theory that has been used to conceptualize how teachers influence student development is Bronfrenner's bioecological 
model (Bronfenbrenner \& Morris, 2006). This theory describes the levels, or ecological systems that interact with and influence development, including the individual level, microsystem, mesosystem, exosystem, macrosystem, and chronosystem (Bronfenbrenner, 1994). Proximal processes are a key concept in this theory and are defined as reciprocal interactions of an individual with the systems in which the individual spends a great deal of time (Bronfenbrenner \& Ceci, 1994; Tudge, Mokrova, Hatfield, \& Karnik, 2009). These increasingly complex proximal processes influence the expression of underlying biological mechanisms thereby contributing to child development (Tudge et al., 2009).

The school context is one of these main systems where proximal processes occur outside of a child's home (Bronfenbrenner \& Morris, 2006). The shift to school is one in which children learn through interacting with others outside of their immediate families, often for the first time (Rimm-Kaufman \& Pianta, 2000). The teacher is a key figure in the school microsystem and through interactions with each student as well as managing student-student interactions, the teacher contributes to child development (Davis, 2003). The teacher's personal wellbeing and emotional states contribute to these individual interactions with students, and over time contribute to the strength of the teacher-student relationship (Jennings \& Greenberg, 2009). Consistent with this theory, a recent study by McLean and Connor (2015) found that teacher depressive symptoms were associated with lower student math achievement at the end of the academic year. Also relating to the processes occurring over time, this study found and that the relationship between teacher depressive symptoms and student achievement was mediated by the quality of the classroom learning environment, which aligns with the theory that moment-to-moment classroom interactions between the teacher and student contribute to long term 
development over time, and are influenced by the teacher wellbeing (McLean \& Connor, 2015).

Attachment Theory. In addition to ecological models describing the influence of teachers on student development, attachment theory has also been used to conceptualize the teacher-student relationship (Pianta \& Steinberg, 1992). Attachment theory describes the early relationships between children and their caregivers, and how these interactions create internal working models that influence social development and future patterns of relating to others (Ainsworth, 1979; Bowlby, 1969, 1988). Secure attachment is characterized by emotional closeness and security for a child to feel comfortable to explore and learn in his or her environment (Bretherton, 1985). Good attachment figures are responsive, consistent, warm, and predictable (Ainsworth, 1979). This theory was first used to describe parent-child relationships, and has then been applied to student interactions with teachers, as similar qualities of the parent-child relationships can be observed between students and teachers (Davis, 2003; Pianta \& Steinberg, 1992). Similar to the ways in which parent-child relationships predict relationships throughout development by the creation of internal working models, teacher-student relationships early in elementary school also predict student academic and behavioral outcomes in adolescence (Hamre \& Pianta, 2001). Consistent with this theory, a meta-analysis of research on teacher-student relationship found a medium effect size between positive relationships with teachers and student engagement $(r=.34)$, a negative association between negative teacher relationships and student engagement $(r=-.32)$, and a small association between positive teacher-student relationships and student achievement ( $r$ $=.16)$. In addition to the teacher-student relationships as important for academic 
engagement and achievement, positive attachment with a teacher may also be protective and buffer against the negative effects of insecure parent-child attachment (Buyse, Verschueren, \& Doumen, 2011).

A caregiver or teachers' emotional factors influence his or her abilities to be consistently warm, and supportive, which are important relational qualitiesthat contribute to secure attachment (Davis, 2003). If a teacher is stressed or experiencing burnout, he or she is more likely to be reactive and unpredictable and use harsh ineffective forms of behavior management (Reinke, Herman, \& Stormont, 2013), which over time have a negative impact on the teacher-student relationship and classroom climate creating a negative environment for learning. Jennings and Greenberg (2009) describe the relationship between teacher stress and the negative impact on student behaviors as a "burnout cascade" in which disruptive student behaviors contribute to increased stress and strain, and teachers then use ineffective forms discipline such as yelling, which exacerbate student behaviors. A student who has a harsh teacher and is in an unpredictable classroom environment may be less likely to take important learning opportunities, such as participating in class. A recent study by Herman et al. (2018) supported this theory and found increased student disruptive behaviors, fewer prosocial behaviors, and reduced student achievement in classrooms with teachers who reported having high stress and were not coping with their stress. High teacher stress and burnout has also been associated with higher levels of salivary cortisol levels of students, which is a physiological indicator of stress (Oberle \& Schonert-Reichl, 2016). Overall this theory and supporting research suggest that teacher-student relationships are crucial to student 
development, and teacher emotional factors can influence the quality of interactions and long-term relationships with students.

\section{Impact of Stress on Teachers}

In additional to impacting student development, high occupational stress over time is associated with negative outcomes for teachers. The first notable negative outcome for teachers is professional burnout experienced by workers in helping professions. Burnout results from prolonged stress and consists of three components: emotional exhaustion, depersonalization, and reduced personal accomplishment (Maslach, Jackson, \& Leiter, 1986). Emotional exhaustion is the main experience of burnout and is defined as the feeling of intense fatigue as a result of working in an emotionally taxing profession (Maslach, Schaufeli, \& Leiter, 2001). Depersonalization describes the experience of disconnection and distancing that occurs as a result of exhaustion. Depersonalization can be viewed as a way to cope with the high stress, by protecting one's emotional resources (Montgomery \& Rupp, 2005); however, depersonalization can lead to becoming jaded and increased harsh interactions with others (Maslach et al., 2001). Reduced personal accomplishment is the third component of burnout and is defined by lacking enjoyment, meaning, and satisfaction from the work. Teachers often enter the education field because they enjoy working with children and want to make a positive impact on youth. With this strong motivation, teachers may overcommit and have a hard time setting limits on their work life, leading to eventual burnout and frustration.

Burnout and high stress are concerning for teachers as they are associated with a host of negative physiological, psychological, and social outcomes. Chronic stress in 
teachers is associated with psychological conditions including an increased risk for anxiety (Steinhardt, Smith Jaggars, Faulk, \& Gloria, 2011) and burnout predicts depressive symptoms in teachers (Shin et al., 2013). Burnout and chronic stress also take a physical toll on the body, as the high presence of stress hormones can impact important biological processes, including the immune system, metabolism, sleep-wake cycles, reproduction, and development (Bellingrath, Weigl, \& Kudielka, 2009; Chrousos, 2009). As a result, stress and burnout are associated with increased risk for cardiovascular diseases, obesity, Type 2 diabetes, and sleep disturbances (Melamed, Shirom, Toker, Berliner, \& Shapira, 2006). Burnout is also associated with risky behaviors including heavy drinking and with lower engagement in healthy behaviors like physical activity (Ahola et al., 2012). In addition to physical and psychological outcomes, there is a social cost to the experience of occupational stress and burnout as it is also related to disconnection from family members and marriage problems (Jackson \& Maslach, 1982). Given the large number of teachers working in the United States, chronic stress and burnout in the field is likely to have a cumulative cost to society, in addition to the negative outcomes on individuals.

\section{Impact of Teacher Stress on the Educational System}

Teacher stress and burnout create problems for the broader educational system, such as absenteeism, turnover, and attrition, and these problems can be very expensive. With the negative health and psychological effects of burnout, teachers who are experiencing high stress are more likely to use sick days and take time off to recover (Darr \& Johns, 2008), and this can be challenging for schools to manage with hiring substitutes and covering for missing teachers. Job performance is also diminished when 
workers are emotionally exhausted (Cropanzano et al., 2003). As a result for teachers, there is a negative relationship between student achievement and burnout (Arens \& Morin, 2016), which can be detrimental to schools when funding is driven by student achievement.

Teacher attrition is especially a critical concern to the educational system as it has a costly impact (Watlington et al., 2010). Barnes et al. (2007) estimated that depending on the area of the school district and size of the school, the cost of one teacher leaving the district ranged from $\$ 4,400$ to $\$ 18,000$ with the cost to recruit, hire and prepare new teachers. This cost compounds in large districts, for example, the yearly cost of teacher turnover in Chicago Public School is estimated to be \$86 million dollars (Barnes et al., 2007). Of additional concern, the rates of turnover are higher in schools with greater need with students from low socio-economic resources (Simon \& Johnson, 2015). Further, the schools with the lowest student achievement levels tend to have the highest rates of teacher turnover (Ronfeldt, Loeb, \& Wyckoff, 2013). This leads to a vicious cycle of the rapid changing of unprepared teachers teaching students with the highest academic needs (Barnes et al., 2007). Working conditions and high stress are often given as reasons for teachers moving to a different district or leaving the field (Ingersoll \& Smith, 2003; Simon \& Johnson, 2015); this is important from a prevention perspective as these constructs may be malleable and targets for intervention to have a positive effect on the broader educational system.

\section{Existing Teacher Stress Reduction Programs}

There are existing programs to support teachers to cope with stress; however, recent programs in stress management for teachers have some limitations in their broad 
appeal, breadth of possible strategies, and length of interventions. Overall, general occupational stress management programs have a stronger empirical support base, and cognitive-behavioral interventions consistently demonstrate positive outcomes for workers in comparison to other types of stress management interventions, including support groups and relaxation training (van der Klink, Blonk, Schene, \& van Dijk, 2001). A meta-analysis by Richardson and Rothstein (2008) of stress management programs for occupational stress found an overall medium to large effect size $(d=0.53)$ and further found that cognitive-behavioral programs had a larger effect on stress reduction $(d=$ 1.16) in comparison to other types of stress training. The relaxation programs had a medium effect size $(d=0.50)$; which is similar to the effect size found in a separate metaanalysis of mindfulness interventions for stress reduction $(d=.49)$ (Grossman, Niemann, Schmidt, \& Walach, 2004). Richardson and Rothstein (2008) also found that shorter programs with fewer components were more likely to have positive effects than more complicated programs. These findings on general occupational stress training can inform the development of stress management programs for teachers, and suggest that the cognitive-behavioral framework is beneficial for stress reduction.

\section{Mindfulness Based Programs}

Despite the positive effects of cognitive-behavioral programs for occupational stress, mindfulness based programs have become increasingly popular for stress management (Sharma \& Rush, 2014). Mindfulness involves deliberate present-moment, and non-judgmental awareness and acceptance of one's experience of thoughts and emotions (Kabat-Zinn, 2003). Through mindfulness-based programs, participants are taught to have greater awareness and self-compassion of their present emotional states 
and this awareness can be beneficial for applying self-regulation strategies. Some mindfulness programs for teachers are also designed to increase teachers' social emotional competencies so that teachers can effectively model and lead social emotional instruction for students (Jennings \& Greenberg, 2009).

The Stress Management and Relaxation Techniques (SMART) in Education program (SMART; Cullen \& Wallace, 2010) is an example of a mindfulness-based training for students, teachers, and other school personnel. Roeser et al. (2013) completed a randomized control trial of this program with a sample of 110 self-selected teachers in the United States and Canada. The program consisted of 36 hours of training over the course of 8 weeks involving direct instruction, group discussion and practice, homework, and journaling (Jennings \& DeMauro, 2017). Through the program teachers learned to develop a greater awareness of their emotional states, the function of emotions, how to identify emotional triggers in the classroom, meditation practices, and self-compassion (Benn, Akiva, Arel, \& Roeser, 2012). The intervention had positive effects on teacher self-reported outcomes, including higher mindfulness, self-compassion, working memory (only assessed in the Canadian teachers), and lower stress, burnout, depression and anxiety after the intervention and at the 3-month follow up; however, there were no intervention effects on physiological indicators of stress including heart rate and salivary cortisol (Roeser et al., 2013).

Another mindfulness-based stress management program is the Cultivating Awareness and Resilience in Education (CARE) for teachers designed to reduce occupational stress as well as improve teachers' abilities to promote social emotional learning in their classrooms (Jennings \& DeMauro, 2017). The CARE program is based 
in the prosocial classroom model by Jennings and Greenberg (2009), in which teacher social-emotional functioning is critical to the classroom climate and promoting positive social emotional development in students. There are three main components of the CARE program: psychoeducation about the purpose and awareness of emotions, mindfulness and bodily relaxation skills and practice, and compassion practice for self-reflection and listening to others (Jennings et al., 2013). The program is intensive in that it involves over 30 hours of training during four-full day trainings with on-going training over the course of four to six weeks. An initial randomized control trial with 50 teachers showed that the intervention had some positive effects on self-reported teacher efficacy, physical symptoms of stress, and the general exhaustion component of burnout (but not on the PANAS, or other indicators of burnout); though researchers did not account for teachers nested within schools, which could have impacted the results. A larger randomized control trial with 226 teachers in New York City examined the effects of the CARE program on teacher-reported outcomes as well as on student outcomes and classroom interactions observed using the CLASS measure (Jennings et al., 2017; Jennings \& DeMauro, 2017). This was the first study evaluating teacher stress programs that accounted for the nesting of teachers within schools using multilevel modeling(Jennings \& DeMauro, 2017). The CARE intervention had small effects on mindfulness $(d=.28)$, psychological distress $(d=-0.18)$, time urgency $(d=-.20)$, and no effects on teaching efficacy. The CARE intervention also had effects on the observed dimension of emotional support provided by teachers to students in their classrooms $(d=.22)$. Despite the positive effects of the intervention, the effect sizes were modest in comparison to 
those in the SMART intervention efficacy trial (Jennings et al., 2017; Roeser et al., 2013).

Mindfulness programs are promising, but the results from initial efficacy trials indicate some limitations. The first limitation is that the largest evaluation of the mindfulness program has small effect sizes, with larger effects on outcomes directly related to mindfulness (Jennings et al., 2017). A possible explanation for the small effects on teacher outcomes is the program is designed to improve social-emotional awareness, regulation, mindfulness and self-compassion, which may support a teacher to calm down in a stressful encounter, but does not seem to sufficiently address other possible sources of stress, especially classroom management. Using the teacher coping categories defined by Kyriacou (2001), these programs seem to emphasize palliative techniques to cope with stress, but may be lacking in direct action techniques which address the source of stress and can prevent the stressors from worsening in the future.

A second possible limitation of mindfulness based programs is that while they may be beneficial for some teachers, it may be limiting to as universal programs in schools, as there is variability in the individual effects of mindfulness programs. Researchers of mindfulness based programs have noted the possibility of self-selection bias, as it may be that mindfulness programs are beneficial to people who decide to enroll in such programs because they have a pre-existing positive perspective of mindfulness (Jennings \& DeMauro, 2017; Sharma \& Rush, 2014), but this may not be the case if the program is used as a universal intervention. Some individuals really enjoy and experience many benefits from mindfulness interventions; but it may be limiting to have only one type of intervention to teachers to cope with stress, as opposed to providing teachers with 
a variety of skills in order to reach a wider range of teachers. This concern of limited teacher desirability might have impacted the recruitment for the large randomized controlled trial of the CARE program in which of 525 teachers who were eligible to participate in the program, less than half (43\%) enrolled in the program, and an additional 6\% did not complete the program (Jennings et al., 2017).

A third limitation of mindfulness based programs is the small number of studies evaluating the efficacy of these interventions for occupational stress management in comparison to other strategies. A meta-analysis of mindfulness programs for stress management found 17 studies between 2009 and 2014, but many studies had small sample sizes and only two had a randomized control design (Sharma \& Rush, 2014). In summary, mindfulness based programs for teachers seem promising for some teachers, but may not be sufficient to reach all teachers, or address the many sources of stress that teachers experience, and further research into the efficacy and effectiveness of these programs is needed.

\section{Cognitive-Behavioral Programs}

In comparison to the more recent research into mindfulness based programs, cognitive-behavioral programs for stress management are based in the combined cognitive and behavioral psychological theories and therapy techniques, which have one of the strongest evidence-bases in comparison to other forms of treatment of psychological distress (Hofmann, Asnaani, Vonk, Sawyer, \& Fang, 2012). The cognitive triangle, or cognitive mediation model is the basic premise for these interventions, and this is the theory that thoughts influence the perceptions of an event and influence a person's behavioral response to an event (Beck \& Beck, 1995). Thus, in order to change 
one's emotional states, a person must change his or her thoughts, and/or change his or her behaviors. The development of skills and practical strategies is essential to cognitivebehavioral programs, and direct application and practice is also critical. Cognitive behavioral interventions are generally intended to be short-term and focused on solving problems. Common cognitive behavioral techniques include behavioral activation (increasing participation in rewarding and stimulating activities), cognitive restructuring (noticing, evaluating, and challenging maladaptive thinking patterns), relaxation strategies (slow and controlled breathing), and systematic desensitization (increasingly challenging exposures to feared environments or stimuli in order to reduce anxiety). Cognitive-behavioral programs for occupational stress have demonstrated positive effects greater than other programs (Richardson \& Rothstein, 2008).

Forman (1982) developed a stress management for teachers program based in stress inoculation theory (Meichenbaum, 1977). In stress inoculation theory there are three phases: psychoeducation about stress; learning cognitive behavioral strategies (i.e., slow and controlled breathing and cognitive restructuring), and applied practice (Meichenbaum, 1977). Forman (1982) examined the effects of a stress management training using this model with 12 teachers and the intervention consisted of six 3-hour trainings. Teachers who received the intervention reported lower stress and anxiety levels immediately after the training and continued reductions at the six-week follow up, but no differences in teacher-student classroom interactions were observed in comparing treatment and control teachers (Forman, 1982). Following this study, these researchers completed a study with 60 teachers comparing the effects of the cognitive-behavioral stress management intervention with a classroom management training (Sharp \& Forman, 
1985). Both programs had similar effects in comparison to the control group and were found to reduce teacher reported stress and anxiety, as well as improve observed positive teacher-student interactions (Sharp \& Forman, 1985). Cecil and Forman (1990) compared the effects of the cognitive-behavioral stress management training with a support group for teachers and found that the stress management training more effective in reducing stress.

More recently, researchers in Germany completed a randomized control trial to compare the effects of teacher stress training and classroom management training (Dicke et al., 2015). The sample consisted of 56 pre-service teachers, as new teachers are particularly at risk for high stress and burnout $(n=22,24$, and 10 for the classroom management, stress management, and classroom management groups, respectively). They found similar positive effects of the stress management training and the classroom management training on teacher-reported well-being and goal achievement in comparison to the control condition (Dicke et al., 2015). In comparing the effects of the stress management training to the classroom management training, the teachers who received the classroom management training reported lower emotional exhaustion and rumination, as well as higher teaching self-efficacy and these effects remained over the year follow up. These results suggest that classroom management may be a critical component in reducing stress and preventing burnout. A stress management program may result in improved coping, whereas the classroom management training may have reduced stress by directly decreasing stress due to handling difficult student behaviors.

Summary of Teacher Stress Interventions Effects. A recent meta-analysis by von der Embse, Ryan, Gibbs, and Mankin (2019) examined published studies of teacher 
stress interventions. Overall they found three categories of programs based in psychoeducation, cognitive-behavioral therapy, and mindfulness. The effect sizes of these programs varied widely (ranging from 0.03 to 2.66 ) because of the differing intervention components, time commitments, and study measures. The authors summarized the findings that generally programs had small to medium effect sizes $(0.2$ to 0.5$)$ and medium to large effect sizes for programs that included a component to address student behaviors (0.5 to 1$)$. Although the trend in published articles on teacher stress interventions suggest that mindfulness programs are increasing in popularity, the effectiveness of these programs varies greatly and there is not clear evidence to suggest that these programs are more effective than other programs in supporting teachers to cope with stress (von der Embse et al., 2019).

\section{Bibliotherapy Interventions}

Relevant to the existing study, while the previously reviewed teacher stress interventions all involve multiple live sessions, altering the delivery method of the intervention may increase the ease and convenience of the program for teachers. One promising method is providing a coping skills intervention through bibliotherapy, or by having teacher read a book that reviews strategies and gives guidance for making changes. Previous researchers have found reductions in symptoms as a result of selfdirected book interventions for participants with mild to moderate depression and anxiety. For example a meta-analysis by Cuijpers (1998) found a cognitive behavioral bibliotherapy intervention for depression had comparable effects sizes to other treatments for depression in therapeutic settings (ranging from 0.65 to 1.21 effect size for the bibliotherapy). Specific to occupational stress, a randomized trial compared the efficacy 
of in-person treatment, phone treatment, and bibliotherapy and found all of the programs reduced occupational stress (Kilfedder et al., 2010). Further there were no statistically significance differences in treatment effects across the types of treatment delivery. One previous study examined a bibliotherapy intervention based in Acceptance and Commitment Therapy (ACT) in a sample of 236 teachers (Jeffcoat \& Hayes, 2012). The program was administered over the 8 weeks and demonstrated reductions in stress, depression and anxiety with effect sizes ranging from 0.48 to 0.98 (Jeffcoat \& Hayes, 2012). These studies suggest bibliotherapy may be a time and cost effective way to provide therapeutic or preventative treatments, and an attractive option for busy teachers.

\section{Gaps in Existing Literature}

Despite the promising effects of existing stress management programs for teachers, further development is needed. Cognitive behavioral strategies have shown positive effects, but recent work in teacher stress has focused more on mindfulness based strategies, which may not be sufficient to address a variety of stressors or reach all teachers in a school as a universal intervention. Further, the prior interventions reviewed all require a time commitment ranging from 19 to 36 hours of in-person meetings and additional assignments outside of the sessions, and this time investment may not be feasible for many teachers.

Building on prior research, Herman and Reinke (2015) developed a brief bibliotherapy program for teachers that is based in the cognitive-behavioral framework and includes components of mindfulness and classroom management within the intervention. This program is promising because the comprehensive framework for coping based in psychological science gives teachers a variety of possible coping 
strategies including direct action and palliative techniques. The intervention is also brief and flexible for teachers in comparison to existing programs, as it primarily consists of bibliotherapy with only one in-person meeting, and online webinars for practice that can be conveniently accessed from home. While this program is based in psychological theory with a strong evidence-base, the effects of the Stress Management Training for Teachers has not yet been evaluated through a randomized control trial. An efficacy trial is the important first step in the research process to determine if a program is an evidence-based intervention (Gottfredson et al., 2015).

The second gap in the literature of teacher stress programs is that existing studies examining stress management programs have not examined changes over time in response to the intervention (Jennings et al., 2017; Sharp \& Forman, 1985). This is an important area recommended for further research as in addition to determining the effects of the program on teacher outcomes, it is also critical to examine potential mechanisms for changes in order to refine and improve upon existing programs (Gottfredson et al., 2015). The essential components of the interventions are important to understand in order to have time and cost-efficient programs for teachers.

Another gap in the literature is that few studies of teacher stress management have examined the effect of programs on students of teachers who participate in the programs. As reviewed previously, teacher stress is associated with student behavioral, emotional, and academic outcomes, so it is likely that programs to reduce teacher stress will be beneficial to students, but these distal outcomes of the stress management program have not been examined (Jennings \& DeMauro, 2017). 
A notable methodological gap in the literature is failing to account for the nesting of teachers within schools when examining the effects of the intervention. Though a prior study found that schools accounts for a small percentage of the variance in teacher burnout (Klusmann, Kunter, Trautwein, Lüdtke, \& Baumert, 2008), it is still be important to control for the clustering of teachers within schools as a first check. Recent evaluations of the mindfulness-based training and cognitive-behavioral interventions have failed to report multilevel considerations in their analyses (Dicke et al., 2015; Jennings et al., 2013).

\section{Present Study}

In considering these important gaps in the literature and following the recommendations of these prior researchers of teacher stress trainings, the proposed study will contribute to existing research in several important ways. First, the model of the stress management training in the present study is unique because it is based in the cognitive behavioral framework, which is associated with the strongest positive outcomes in occupational stress management programs (Richardson \& Rothstein, 2008). While grounded in this theory, there are also modules related to classroom management (Dicke et al., 2015; Sharp \& Forman, 1985) and mindfulness, which have been found to be effective strategies for reducing teacher stress.

The second way this study furthers existing research is it evaluates a program that requires much less of a time and cost investment from teachers and schools. Effective and efficient programs are critical for teachers who may already experience high levels of stress. The intervention is flexible as the majority of the intervention can be completed at home through reading the book or participating in online webinars. Though there have 
been prior studies regarding the efficacy of bibliotherapy programs Cuijpers (1998), few of these stress management programs have been widely disseminated to teachers. The intervention is may be more convenient for teachers, and has greater potential for scalability and dissemination.

The third way this study furthers existing research is by examining the change that possibly leads to reductions in teacher stress as a result of the program. Prior studies have examined the effects by comparing pre-and post-measures, but none have examined changes over time, which are critical to examine in program evaluation research (Gottfredson et al., 2015). 


\section{CHAPTER III: METHODS}

This section will include an overview of the study methods. First, it will outline information about the participants. Second, information about each of the self-report measures and observational measures will be reviewed. Third, study procedures will be described. Finally, there will be a description of the data analytic plan.

\section{Participants}

\section{Sample}

Initially the proposed a sample was for 100 teachers in the study with an equal number of teachers in the intervention and in the waitlist control condition. A small effect size of .4 was used to calculate the minimum sample size needed to have sufficient power to detect an effect of classroom observations. A previous study examining the variation in teacher exhaustion that can be attributable to the school level found that it was very small and around $1 \%$ (Klusmann et al., 2008). Since the intraclass correlation may be low for teachers nested within school and exhaustion effects, the power analysis for this study was based on a simple individual random assignment design. Using the Powerup! spreadsheet, with a set alpha level of .05 , statistical power of 0.8 , a two-tailed test, an $\mathrm{R}^{2}$ of 0.5 , the estimated sample size needed to detect an effect size of 0.4 is 100 teachers (Dong \& Maynard, 2013).

Though the proposed sample was 100 teachers, due to difficulties with recruitment, only a total of 53 teachers participated in the study. The challenges with recruitment are reviewed in the limitation section (page 52). There were two separate phases of recruitment of the study in order to increase the number of participants. During the first phase of data collection in winter of 2019, 16 teachers participated and in the 
second phase of the study in fall of 2019, 36 teachers participated. Twenty-six teachers were assigned to the treatment condition and 27 teachers were initially assigned to receive the waitlist control. One teacher in the control group dropped out in the middle of the study, because they left the profession.

Inclusion and Exclusion Criteria. In order to generalize the results of this study, there were few restrictions on participation in the study. Teachers in K-12 classrooms were invited to participate. They needed to have access to the Internet in order to complete study measures and online components of the intervention. Preservice teachers were excluded from the study. Initially, teachers from special education classes were excluded from participation due to differences in classroom structure that could make it difficult to compare classroom observations; however, this criterion was changed based on feedback from special education teachers requesting support for stress management.

Sample Characteristics. Of the teachers who participated in the study, $35.8 \%$ taught elementary school, $26.4 \%$ taught middle school and $37.7 \%$ taught high school. Teachers were from 6 school districts in the middle of Missouri ranging from 3 to 22 teachers per district. There were teachers from 26 different schools participating in the study. The average number of teacher participants per school was 2; 16 schools had only 1 teacher participate.

Teacher demographic information comparing the treatment and waitlist control conditions are presented in Table 1 (see Appendix E). Of the participants, $84.9 \%$ identified as female and $15.1 \%$ identified as male; $92.5 \% 5$ of the teachers identified as White, $3.8 \%$ identified as Asian, $1.9 \%$ identified as Black and 1.9\% identified as multiracial. Teachers ranged in age from 21 to 58 years old $(\mathrm{M}=37.70 \mathrm{SD}=11.13)$. 
They ranged in years of teaching experience from 0 (teachers in their first year of the profession) to 33 with an average of 10.28 years of experience ( $\mathrm{SD}=9.38)$. In the sample, $49.1 \%$ of teachers had a bachelor's degree as their highest level of education and $51 \%$ had a master's degree.

\section{Measures}

Teachers completed two self-report surveys consisting of measures of stress, coping and related constructs. The measures were completed during the baseline data collection and then at the follow-up time point. Trained observers also completed two 20minutes classroom observations in teachers' classrooms. Additionally, teachers reported on their weekly use of coping strategies through a brief self-report survey through the course of four weeks. The scale information including alpha and omega reliability, mean, and standard deviation for study measures is presented in Table 2 (see Appendix E).

\section{Self-Report Measures}

Stress and Coping Items (Herman \& Reinke, 2012). The stress and coping items were used to measure overall perceptions of teacher stress and their awareness, use, and understanding of coping strategies. Items ask about overall perceptions of stress and coping, as well as stress and coping in the past week. Teachers are asked to respond on an 11-point Likert scale ranging from 0 to 10. Sample items include "How stressful is your job overall?" ranging from 0 (not stressful) to 10 (very stressful), and "How well are you coping with the stress of your job right now?" ranging from 0 (not well) to 10 (very well). While traditional reliability measurements cannot be calculated with single-item measures, these items have demonstrated concurrent and predictive validity for teacher self-reported stress, as well as sensitivity to change to intervention effects (Eddy, 
Herman, \& Reinke, 2017). Similar single-item stress measures were also used by stress researchers (Forman, 1982).

Teacher Stress Inventory (TSI; Fimian, 1984). The Teacher Stress Inventory (TSI) is a 49-item measure of stress specifically for teachers. It includes subscales related to common sources of teacher stress including time management, discipline and motivation of students, and professional investment. It also includes subscales related to emotional, behavioral, and physiological experiences of stress. It has demonstrated concurrent, predictive and factorial validity in a population of teachers in the United States (Fimian \& Fastenau, 1990). This scale was also used to evaluate a teacher stress management program in prior studies (Cecil \& Forman, 1990). Participants respond on a 5-point Likert scale with responses ranging from 1 (not noticeable/no strength) to 5 (extremely noticeable/major strength). Sample items include: “There isn't enough time to get things done," and "I respond to stress by sleeping more than usual." The alpha and omega reliability estimates for this measure ranged from .89 to .90 .

Perceived Stress Scale (PSS; S. Cohen, Kamarck, \& Mermelstein, 1983). The Perceived Stress Scale is a broad measure of stress. It includes ten items related to an individual's experience with stress in the past month on a five-point Likert scale ranging from 0 (never) to 4 (very often). Sample items include: "In the last month, how often have you felt that you were unable to control the important things in your life?" "In the last month, how often have you been able to control irritations in your life?" The PSS has been used to evaluate stress trainings for teachers in previous studies (Roeser et al., 2013). In the current study, the reliability estimates for this measure ranged from .86 to .88 . 
Brief COPE (Brief COPE; Carver, 1997). The Brief COPE is a short version of a scale measuring common coping strategies used to handle stress (Carver et al., 1989). For this study, the measure includes 23 items of possible behaviors related to different types of coping responses, such as avoidance, denial, and problem solving. The response options range from 1 (I haven't been doing this at all) to 4 (I've been doing this a lot). Sample items include: "9. I've been trying to see it in a different light, to make it seem more positive," and "I've been blaming myself for things that happened." Items on the scale are summed to create brief subscales about specific coping behaviors (e.g., positive reframing, use of humor). These subscales included 2 items each and the substance use was a single item. Most of the reliability estimates in this sample were about the recommended alpha level of .7 at pre and post measurement time points. The selfdistraction and venting subscales had alpha and omega values below .7, ranging from .52 to .56 for self-distraction and .56 and .64 for venting.

Maslach Burnout Inventory (MBI; Maslach et al., 1986). The Maslach Burnout Inventory was used to measure symptoms of teacher burnout. This scale is made up of three subscales measuring emotional exhaustion, depersonalization, and personal accomplishment. The MBI consists of 22 items with a 7-point Likert scale ranging from 0 (Never) to 6 (Every day). Sample items include: "I've become more callous toward people since I took this job." and "I have accomplished many worthwhile things in this job." Reliability estimates of the three subscales range from Cronbach's alpha of .76 to .9 (Maslach et al., 1986) and independent researchers have examined concurrent and factorial validity of this measure for use for teacher-reported burnout (Byrne, 1993). In the current study, the reliability estimates for the subscales ranged from .77 to .91 . 
Teacher Sense of Self-Efficacy Scale (TSES; Tschannen-Moran \& Hoy, 2001). This measure of teacher reported self-efficacy has demonstrated reliability in a sample of teachers in the United States (Cronbach's alpha ranging from .76 to .87) as well as concurrent and factorial validity (Klassen et al., 2009). The 12-item short-form of this scale was used for the study with a 9-point Likert scale ranging from 1 (None at all) to 9 (A great deal). The reliability estimates of alpha and omega ranged from .85 to .94 in this study.

Organizational Health Inventory(OHI; Hoy \& Fedman, 1987). Two subscales from the Organizational Health Inventory for Elementary Schools were used in this study. The subscales included are the collegial leadership subscale (10 items) and the teacher affiliation subscale ( 9 items). The responses range from 0 (rarely occurs) to 4 (very frequently occurs). Sample items include: "The principal goes out of his or her way to show appreciation to teachers," and "There is a feeling of trust and confidence among the staff." Each subscale demonstrated acceptable reliability with alpha and omega estimates ranging from .85 to .89 for teacher affiliation and from .94 to .96 for collegial leadership.

Teaching Satisfaction Scale (TSS; Ho \& Au, 2006).Teachers responded to a fiveitem scale measuring their job satisfaction on a 5-point Likert scale ranging from 1(strongly disagree) to 4 (strongly agree). The scale has items related to overall job satisfaction such as, "in most ways, being a teacher is close to my idea," "I am satisfied with being a teacher," and "If I could choose my career over, I would change almost nothing." The TSS has been used in other studies of teacher job satisfaction and has demonstrated adequate reliability and validity evidence (Duffy \& Lent, 2009; Ho \& Au, 
2006). The reliability estimates for this scale ranged from .87 to .88 in this sample of teachers.

Patient Health Questionnaire - 8 (PHQ-8; Kroenke et al., 2009). The PHQ-8 is a common screener for depressive symptoms. The PHQ-8 is based off of the PHQ-9, but does not include an item about suicidality. In a meta-analysis of studies with the PHQ-9 summarizing data from more than 32,000 patients in a primary care setting the measure demonstrated sensitivity of .80 and specificity of .92 in screening for depression (Gilbody, Richards, Brealey, \& Hewitt, 2007). On the PHQ-9, 10 is used as the cutpoint for screening for clinical depression, or scores of 5, 10, and 15 indicate severity levels of mild, moderate, and severe depression (Kroenke, Spitzer, Williams, \& Lowe, 2010). The PHQ-8 is a brief 8-item measure on a 4-point Likert scale ranging from 0 (not at all) to 3 (nearly every day). Participants are asked to respond to their experience of depressive symptoms in the last 2 weeks. Sample items include: "Over the last 2 weeks, how often have you been bothered by any of the following: little interest or pleasure in doing things?; Trouble falling or staying asleep, or sleeping too much?” In the current sample, the alpha and omega reliability estimates ranged from .87 to .90 .

Generalized Anxiety Disorder-7 (GAD-7; Spitzer, Kroenke, Williams, \& Löwe, 2006)The GAD-7 is a brief scale that asks about general anxiety symptoms on a 4-point Likert scale that ranges from 0 (not at all) to 3 (nearly every day). In a sample of 2,740 patients, the GAD-7 had a sensitity of .89 and specificity of .82 in screening for generalized anxiety disorder (Kroenke et al., 2010). Similar to the PHQ-9, a cutpoint of 10 is used to detect clinically significant anxiety, and 5,10,15 suggest anxiety severity levels of mild, moderate, and severe. Sample items include: "Over the last 2 weeks, how 
often have you been bothered by any of the following: Feeling nervous, anxious or on edge? Not being able to stop or control worrying?". In the current sample, the reliability estimates ranged from .87 to .90 at the pre and post time points.

Weekly Self-Monitoring. All teachers in the study also completed four weekly reports of stress and coping strategies that will take about five minutes or less to complete (Appendix B.). Teachers completed the Stress and Coping Items (Herman \& Reinke, 2012), and they will also self-reported their use of common coping strategies. Teachers received links to complete these surveys through email.

\section{Observation Measures}

In addition to online surveys, trained data collectors completed one classroom observation for each teacher prior to intervention and one observation post intervention. Each observation was 20 minutes, and did not disrupt typical instruction. Observers coded specific teacher behaviors as well as completed global ratings of the classroom. Previous studies into the efficacy of teacher stress programs have used similar observations to see if the program has an effect on teacher-student interactions (Cecil \& Forman, 1990; Forman, 1982; Sharp \& Forman, 1985).

Brief Classroom Interaction Observation-Revised (BCIO; Reinke \& Newcomer, 2010). The BCIO is a brief observational code for trained observers to capture the interactions between teachers and students in the classroom using electronic devices with the Multi-Option Observation System for Experimental Studies software (MOOSES; Tapp, 2004). Observers who conducted the observations received training and on-going reliability checks with inter-rater reliability of $80 \%$ or above. 
Trained observers completed two additional observational forms. The Classroom Atmosphere Rating Scale (Conduct Problems Prevention Research Group) and the Overall Rating Form were completed after twenty-minute observations of the classroom climate. Observers rated teachers' use of classroom management strategies including reinforcement, supervision, review of expectations, and responsiveness, as well as overall student responses including compliance, interest, and on-task behaviors.

\section{Procedures}

\section{Recruitment}

Following approval from the Institutional Review Boards with the University of Missouri as well as with the local school district, the investigators presented the study to local administrators and principals to inform them of the study and requested to complete recruitment in their schools. Then recruitment efforts occurred over email and in-person in the schools. The principal investigator emailed all teachers in the district to inform them of the study. Some principals also had the representatives from the study recruit teachers to participate during team meetings and during professional development trainings for teachers new to the district.

Teachers were recruited from the local area initially, but then recruitment efforts expanded to the surrounding districts to maximize participation. The school district who had the most participants take part in the study also offered their teachers professional development credit for taking part in the study.

Consent. Teachers who were interested in the study gave written consent to the study procedures. Teachers agreed to random assignment and to keep the identity of other study participants private. Intervention teachers also signed an agreement to keep 
intervention materials confidential during the study. In order to participate, teachers also consented to having data collectors complete two observations in their classrooms. Since the intervention was at the teacher level and no identifying information was collected on students in the classrooms, consent from parents of students was not required by the Institutional Review Boards with the university and the district.

Following recruitment, all teachers participated in baseline data collection consisting of self-report surveys and classroom observations. As previously reviewed, the self-report surveys asked about the following constructs: stress, coping, burnout, depressive symptoms, anxiety symptoms, self-efficacy, wellbeing, and job satisfaction. Teachers received links through emails to take the surveys online. If the teachers did not complete the survey within a week of receiving the survey, a member of the research team followed up with them to send the link again. All baseline and follow up study measures were completed within two weeks. The surveys included about 120 items and took 15-25 minutes to complete. Teacher stress was measured in two ways, as in previous research (e.g., Kyriacou \& Sutcliffe, 1978), with a longer measure of teacher stress as well as global ratings of teacher stress and coping. After the intervention, the teachers completed the same self-report measures and observations (see Appendix A. for the project timeline).

In order to examine the intervention effects over time, teachers were also asked to complete a brief self-report survey of their use of coping strategies (see Appendix B, weekly self-report measure). This measure took about five minutes or less to complete, and teachers were sent the link through their emails in order to receive the survey. 
Teachers completed the weekly self-report during baseline data collection, during four weeks of the intervention, and once during the follow up.

Study Phases. The first phase of the study started in February of 2019 and was completed in April of 2019. The teachers in the waitlist condition of phase 1 completed the intervention in April and early May of 2019. In the first phase of the study, there were 16 teacher participants with 8 in each condition. Individual teachers were randomized to each condition, and block randomization in schools was used to ensure an equivalent distribution of teachers in each condition across schools. The number of participants in the sample was a lower than initially targeted for the first phase, so additional recruitment efforts were completed in the fall of 2019.

Based on feedback from teachers in the first phase of the study, new teachers to the field were targeted for recruitment through professional development trainings in-person. Thirty eight teachers participated in the second phase of the study from September of 2019 to November 2019. Baseline observations and surveys were collected in September and early October, and the intervention was completed in mid-October. Follow-up data collections occurred four to six weeks following the in-person training. Waitlist teachers in phase 2 completed the training in December and early January.

Teacher Compensation. Teachers were compensated for their time to complete the study measures. After completing the baseline self-report measures, teachers were mailed checks for $\$ 25.00$. Teachers were also compensated $\$ 5.00$ for each weekly selfmonitoring form they completed over four weeks, with the potential to receive $\$ 20.00$ in compensation for completing these forms following the intervention. After teachers completed the self-report forms and observations for the follow-up data collection period, 
teachers received an additional $\$ 25.00$ to compensate them for their time. Overall participants received $\$ 70.00$ through the course of the study.

\section{Intervention}

Theory of Intervention Change. The primary purpose of the intervention was to improve teachers' cognitive-behavioral coping. It was hypothesized that by increasing the use of these coping strategies the intervention will have an effect on teacher and student outcomes (see Appendix C, for theory of change). The first part of the intervention increased awareness and understanding of stress and the purpose of stress as a potentially adaptive and helpful emotion. Shifting perceptions of stress to view the emotion as something as normal and even potentially beneficial, has been associated with reductions in burnout (Kim, Shin, Tsukayama, \& Park, 2020). The second part of the training was grounded in cognitive-behavioral theory with the model that thoughts, behaviors, and emotions are all connected (Beck \& Beck, 1995). By changings thoughts and behaviors, it is possible to reduce strong emotions, including stress and anxiety. It is hypothesized that by increasing the use of cognitive-behavioral coping skills, this will help teachers to reduce sources of stress and manage their symptoms of stress. Through these reductions in stressors and improved coping, it is hypothesized that teachers will experience shortterm outcomes of decreased stress and improved teacher-student interactions. Over time, these short-term outcomes are hypothesized to lead to long-term outcomes of improvements in job satisfaction and decreased burnout. As previously reviewed in the measures sections, these constructs were measured throughout the study in order to test this theory of change. 
Intervention Overview. The intervention was a four-week course consisting of one in-person training and three additional online trainings.

In-Person Training (Overview of the Program). The in-person training was 6090 minutes and was given in the local library or at an on-campus location. Teachers participated in a workshop led by a trained member of the research team. The trainer had a standardized script for the training and a PowerPoint presentation with the material from the manual. The format of the training included a combination of didactic instruction, group discussion, self-reflection, and practice. Due to scheduling conflicts, some teachers could not make the in-person trainings, so the trainer met individually with 9 teachers in order to deliver the session content.

The purpose of the training was to review the Stress Management Training for Teachers program. First, the trainer introduced the rationale for providing teachers with stress management training and the promise of bibliotherapy to improve coping with uncomfortable emotions like stress. Second, the program provided teachers with education about the biological purpose of stress as a helpful short-term physiological response to prepare the body for action that can be harmful when prolonged over time. Third, the workshop trainer introduced the Teacher Coping Model, which is the core of the stress management training. Fourth the trainer taught specific strategies for developing awareness of stress, mood monitoring, adaptive thinking, and behavioral strategies to reduce stress. Teachers were assigned a daily mood self-monitoring task to complete during the week, prior to the first online training.

Online training 1 (30 minutes) - Stress Management for Teachers Chapters 5-

6. The purpose of the first online training was to review the chapters in the manual 
related to adaptive thinking. Chapter five reviews a method of tracking positive and negative thinking patterns, and developing adaptive coping thoughts. Chapter six reviews a structured method for addressing common thinking errors and challenging maladaptive thinking patterns. Teachers were assigned to read the two chapters from the manual and to practice one of the cognitive coping methods introduced in the chapters.

\section{Online training 2 (30 minutes) - Stress Management Training for Teachers}

Chapters 7-8. The purpose of the second online training was to review behavioral strategies to cope with teacher stress. Chapter 7 discusses general behavioral strategies including increasing positive activities, problem solving, and relaxation. Chapter 8 reviews classroom behavioral principles and classroom management strategies to increase teachers' self-efficacy and competence to effectively manage classroom behaviors. Dr. Wendy Reinke, one of the creators of the program, led the online video about classroom management. Teachers were assigned to read the two chapters from the book about these strategies, and will be tasked with attempting one behavioral strategy to cope with stress and one strategy related to classroom management.

\section{Online training 3 (30 minutes) - Stress Management for Teachers Chapter 9,}

10, and 14. The purpose of the third online training was to discuss strategies for improving wellbeing, beyond stress management and plan for continued growth. Dr. Herman, one of the creators of the program, led the online training for long-term goal setting. Chapter 9 discusses ways to increase mindfulness as well as connect with exploring one's values. Chapter 10 provides specific examples of specific applications of the manual to common challenges that teachers face (i.e., administrative pressures, and peer conflicts). Chapter 14 concludes with a professional development plan for teachers 
to prepare for continued growth. The third online training prompted teachers to troubleshoot any challenges in applying the material from previous chapters and develop a long-term plan for continued wellness

Intervention Fidelity. Adherence to the program was measured in a few ways. First, attendance for the in-person training was a requirement of the program to ensure that all of the teachers in the intervention received the program content. The teachers also reported on their use of coping strategies during the week through the weekly rating forms of stress and coping. Following the intervention, teachers' reported on their use of the program components of the book, the webinars, and gave quantitative and qualitative feedback on the feasibility and acceptability of the intervention.

\section{Analyses}

Before the analyses were completed, assumptions for multilevel modeling were examined. Multicollinearilty was examined with the variance inflation factors (VIF), and all values were all below 3 (Beck \& Beck, 2015). The percentage of missing data was low; $98 \%$ for self-report baseline and follow-up measures and $94 \%$ for the weekly rating forms had completed data. There were 2 cases for the weekly rating forms who did not complete all of the ratings and these missing cases were handled using listwise deletion. Teachers in the intervention and control were compared using multiple t-tests for all baseline measures to ensure that the groups were relatively similar. The groups were balanced across demographic categories, suggesting that the randomization was successful in creating equivalent groups. There was no statistically significant difference between intervention and control teachers on any of the self-report measures at baseline. There was a statistically significant $(p=.02)$ difference between the two groups on the 
Overall Rating Form sum score. The mean of the intervention group was $34.92(S D=$ 4.26) in comparison to the mean of the control group of $31.85(S D=4.93)$; however, this was mitigated by controlling for baseline in all analyses of treatment effects.

All intervention effects were examined using multilevel modelling in R ( $\mathrm{R}$ Core Team, 2020). The regression analyses were completed using the lme function from the nlme package (nlme: Linear and Nonlinear Mixed Effects Models; Pinheiro, Bates, DebRoy, Sarkar, \& R Core Team, 2020). Multilevel modelling was used to account for clustering of teachers in schools, and fixed effects were included in each model to account for district level variation (represented by $\alpha$ 's in the model). Each model also included Level-1 covariates of the baseline measure and controlled for years of teaching experience. The multilevel models had random intercept and random slopes, allowing these estimates to vary at the school level. The equation for each model had the following format:

Level-1 Model (teacher):

Outcome $_{i j}=\beta_{0 j}+\beta_{1 j} *($ Intervention Status $)+\beta_{2 j} *\left(\right.$ Baseline sum score $\left._{i j}\right)+$ $\beta_{3 j} *($ Teacher Experience $i j)+r_{i j}$

Level-2 Model (school):

$$
\begin{aligned}
& \beta_{0 j}=\left(\alpha_{00}+\alpha_{2} \text { District } 2+\cdots+\alpha_{6} \text { District6 }\right)+u_{0 j} \\
& \beta_{1 j}=\gamma_{10}+u_{1 j} \\
& \beta_{2 j}=\gamma_{20} \\
& \beta_{3 j}=\gamma_{30}
\end{aligned}
$$

All measures were standardized to z-scores. The Benjamini Hochberg method was used to correct for alpha inflation for the multiple comparisons (Benjamini \& 
Hochberg, 1995). As a result of this correction, two intervention effects no longer met the threshold for statistical significance. All models were also rerun to compare results when special education teachers and private school teachers were excluded. There were no statistically significant differences in the intervention effects when these teachers were excluded from the models. The results reported included all cases with complete data. In order to compare with previous studies of teacher stress, effect sizes were also calculated (see Table 8; Cohen, 1988; von der Embse, Ryan, Gibbs, \& Mankin, 2019) using the following formula with the mean difference $(\bar{x})$ comparing post and pre-test scores for the treatment and control groups over the pooled standard deviation:

$$
\begin{gathered}
d_{\text {treatment }- \text { control }}=\frac{\bar{x}_{\text {treatment }}-\bar{x}_{\text {control }}}{S_{\text {pooled }}} \\
S_{\text {pooled }}=\sqrt{\frac{\left(N_{T 2}-1\right) S D_{T 2}^{2}+\left(N_{T 1}-1\right) S D_{T 1}^{2}}{\left(N_{T 2}-1\right)+\left(N_{T 1}-1\right)}}
\end{gathered}
$$

The change in stress and coping over time was also examined using growth modelling in $\mathrm{R}$ ( $\mathrm{R}$ Core Team, 2020) with the lme function from the nlme package (nlme: Linear and Nonlinear Mixed Effects Models; Pinheiro, et al., 2020). These equations had 3 level models with Level 1 - Time, Level 2 - Teacher, and Level 3 - School. The multilevel models estimated random intercepts allowed to vary for the individual teacher and at the school level. Random slopes was not used to reduce the number of parameters to estimate. Figures were plotted using the ggplot function from the ggplot2 package (Wickham, 2016).

Level-1 Model (Time):

$$
\text { Outcome }_{i j}=\pi_{0 j}+\pi_{l j} *(\text { Intervention Status })+e_{i j}
$$


Level-2 Model (Teacher):

$\pi_{0 j}=\beta_{00}+r_{0 j}$

$\pi_{1 j}=\beta_{10}$

Level -3 Model (School):

$\beta_{00}=\gamma_{00}+u_{0 j}$

Finally, as an additional check of the findings, pre-post comparisons were completed with the teachers in the waitlist condition. Multiple t-tests were completed in $\mathrm{R}$ for each of the outcomes and then adjusted with the Benjamini Hochberg method for $p$ value correction. Cohen's d effect sizes were calculated using the formula above. The effect size interpretation is based on the general guidelines of .2 as a small effect, $.5 \mathrm{a}$ medium effect, and .8 or greater a larger effect (Cohen, 1988). 


\section{CHAPTER IV: RESULTS}

\section{Descriptive Statistics}

First, descriptive statistics were completed for demographic information about teachers in the study (Table 1). Second, each measure was analyzed for distribution, skewness, and reliability using Cronbach's alpha (Cronbach, 1951) and omega from the MBESS package (Kelley, 2007) in R (R Core Team, 2020). The scale analytics are presented in Table 2. With the exception of the single-item and 2-item subscales on the Brief Cope, the measures were above the recommended alpha levels of 0.7 , and the interpretation of omega reliability is similar (McNeish, 2018).

\section{Self-report surveys}

The study examined treatment effects on immediate outcomes of stress and coping specifically targeted by the stress management program including overall perceived stress, stress specific to teaching, burnout (Table 3) and coping behaviors (Table 4). The study also examined outcomes on mental health including anxiety and depression (Table 5). The study measured contextual treatment effects of perceived efficacy, organizational health, overall job satisfaction (Table 6), and observed teacherstudent behaviors from independent observations (Table 7). All scales were standardized so the $\beta$ 's reported can be interpreted as standardized effect sizes and for comparison with other studies, Cohen's d effect sizes are also reported.

\section{Results of Intervention Effects}

Teacher Stress and Burnout. From analyses of main effects, teachers in the intervention condition demonstrated a reduction of self-reported stress on the Perceived Stress Scale (Table 3). Teachers in the intervention reported a reduction in their overall 
perceptions of stress in comparison to control teachers and accounting for their baseline stress $\left(\beta_{1 j}=-0.53, p<0.001\right)$. The effect size from the simple pre-post comparison is medium $(d=-0.64)$.

There was no main effect on overall teacher stress from the Teacher Stress Scale which measured classroom level stressors including disruptive behaviors, time management, and physical symptoms of stress. From the Maslach Burnout Inventory, there were no statistically significant main effects on depersonalization, personal accomplishment, or emotional exhaustion.

Teacher Coping. The Brief Cope measure does not have a sum score, and instead subscales can be summed to examine the self-reported coping behaviors (Carver, 1997). The intervention did not have effects on the following subscales: acceptance, humor, instrumental support, religion, use of emotional support, self-blame, self-distraction, and venting. There were main effects for four of the subscales including medium effects for planning $\left(\beta_{1 j}=0.62, p=0.01 ; d=0.66\right)$, positive reframing $\left(\beta_{1 j}=0.66, p=0.01 ; d=\right.$ 0.67), and a small effect for the single-item measure of substance use $\left(\beta_{1 j}=-0.44, p=\right.$ $0.01 ; d=-0.34)$. There was also a significant uncorrected reduction in self-reported active coping $(d=0.41)$; however, this finding was non-significant after adjusting for multiple comparisons.

Mental Health Screening Outcomes. There were main effects of the intervention on the mental health screening measure of anxiety (GAD-7). Teachers in the intervention condition decreased in their self-reported anxiety $\left(\beta_{1 j}=-0.51, p<0.01\right)$ with a medium effect size $(d=-.67)$. The intervention also had a non-significant small to medium effect on the PHQ-8 $\left(\beta_{1 j}=-0.40, p<0.08 ; d=-0.48\right)$. 
In examining the severity levels on these mental health screeners, the cutoffs on the GAD-7 for anxiety symptoms are 5-mild, 10-moderate, and 15-severe, and for depressive symptoms on the adjusted 8-item PHQ are 4.4-mild, 8.8- moderate, and 13.3severe. Categorical variables were created using these cut points. To examine the individual intervention effects, the change in participants' clinical severity categories were examined for depression and anxiety (See Figures 7 and 8). In the control sample ( $n$ $=26)$ the majority of teachers remained in the same clinical severity level $(65.4 \%)$ on the GAD-7, 23.1\% increased, and 11.5\% decreased in severity level. For the GAD-7 in the intervention sample ( $n=26)$ the majority of teachers decreased in clinical severity $(50 \%)$, 46.2\% remained in the same clinical severity level, and 3.8\% in increased in symptom severity. These categorical differences were statistically significant with a 2 by 3 Chisquare analysis (Cramer's $\mathrm{V}=.45, p=.005)$. The effect size interpretation for Cramer's V for 2 degrees of freedom according to Cohen (1988) is a cutoff of .1 for a small effect, .21 for a medium effect, and .35 and greater for a large effect.

On the PHQ-8 in the control sample, the majority of teachers remained at the same clinical level (53.8\%), 34.6\% increased in severity, and $11.5 \%$ improved. For the intervention sample, the majority of teachers remained at the same clinical severity level (50.0\%), $7.7 \%$ increased in severity, and $42.3 \%$ improved. These categorical differences were statistically significant with a 2 by 3 Chi-square analysis (Cramer's $\mathrm{V}=.42, p$ $=.01)$ suggesting a large effect.

Other Self-Report Measures and Observational Measures. The intervention did not have effects on teachers' reported self-efficacy, organizational health scores, or ratings of job satisfaction. There were no main effects of the intervention on any of the 
observed outcomes including the frequency of classroom behaviors recorded on the $\mathrm{BCIO}$ as well as the overall ratings of classroom climate on the CAS and ORF.

\section{Change in Intervention Effects over Time}

Weekly Coping Ratings. The weekly ratings of stress and coping each week demonstrated changes in teacher stress over the course of the intervention. The average ratings on these single-item measures of stress and coping are plotted in Figures 1 to 6 comparing intervention and control teachers. The error bars represent the standard error of the measures. These ratings were completed once during baseline, 3 times weekly during the intervention, and then once more at the follow up time point, 4-6 weeks after the intervention.

The results from growth models examining the change over time are presented in Table 7. There was no change in stress reported on the single-item measure over time and no intervention effect. For the global rating for coping, intervention teachers had a greater rate of change ( $\beta=0.38, p=.01)$. Teachers in the intervention condition also had a greater rate of change for confidence in coping $(\beta=0.39, p=.001)$. Both teachers in the intervention and control condition had statistically significant improvements in their awareness $(\beta=0.36, p=.002)$, understanding $(\beta=0.44, p<.001)$, and use of coping strategies over time $(\beta=0.32, p<.001)$; however the rate of improvement for use of coping strategies was greater for teachers in the intervention $(\beta=0.37, p=.008)$.

Waitlist Condition Reported Stress. The pre- and post-intervention comparisons for teachers in the waitlist control group are presented in Table 9. The means and standard deviations for study measures are reported for Time 1 (baseline), Time 2 (before wait-list received the intervention), and Time 3 ( after wait-list received the 
intervention). Simple t-tests were completed for each measure to examine the difference between Time 2 (the measure completed after the first intervention) and Time 3 . There were statistically significant difference in teacher's reported overall coping $(d=0.44)$, awareness $(d=1.20)$, understanding $(d=0.74)$, and use $(d=0.57)$ of coping strategies. The difference on the Perceived Stress Scale was non-significant with the $p$ value adjustment.

Fidelity to the Intervention. Fidelity to the intervention was measured in a few different ways but was more limited for the independent book and webinar components. All teachers attended an in-person training and received the same dose of the beginning portion of the training. Fidelity to use of the book and the online videos was also measured through a self-report survey where teachers reported their use of these intervention components. From the view counts on the YouTube videos, it is estimated that $1 / 3$ to $1 / 2$ of the intervention teachers completed all of the weekly video assignments. For two of the weekly videos, it was difficult to determine the view count, as participants were given the option to choose between videos, so one participant may have watched both videos, or they could have been separate participants. It is also possible that participants completed the book chapter readings and did not use additional supports from the video.

\section{Feedback on the Intervention}

Acceptability Ratings. Following participation in the teacher stress program, the intervention and waitlist teachers were also asked to evaluate the acceptability of the program. Of the 52 teachers who completed the program, 3 waitlist teachers were not able to schedule a time for the in-person training, but instead elected to participate in an 
upcoming online webinar of the program. An additional 4 waitlist teachers declined to provide feedback on the program, resulting in a final sample of 45 teachers who completed the follow-up program evaluation questions. Teacher were asked about their view of the program overall and the majority (53\%) rated the program as "very positive," $45 \%$ of teachers rated the program as "positive," and $2 \%$ (one teacher) rated the overall program as "neutral."

Participants also provided ratings for individual components of the training including the in-person meeting, the book, and the online videos. For the in-person training, the majority of teachers rated the meeting as "helpful" (51\%) or "very helpful" (40\%); a few teachers reported they were "neutral" about the meeting (6\%), and one teacher $(2 \%)$ reported the meeting was "not helpful." Similarly, the majority of participants found the online trainings helpful (50\%) or very helpful (20\%), and $30 \%$ reported they were neutral about the online components. The book was also well-received by teachers with the majority reporting it was either "helpful" $(42 \%)$ or "very helpful" $(47 \%)$, and a smaller percentage found the book "neutral $(9 \%)$ or "not helpful $(2 \%)$.

Open-ended Feedback. The qualitative data about what teachers liked most and liked least and suggestions for improvement is included (see Appendix F). Some of the themes from the feedback were that teachers enjoyed being able to connect and talk with others and the trainer about stress and by doing so, they didn't feel alone in having stress in the profession. Some teachers reported they liked having new strategies to try out and make small changes over time. Many reported they enjoyed the flexibility of the program and the accessibility of the materials. 
In addition to positive feedback, teachers reported what they liked least about the program (see the Appendix F). The most common barrier was that they had limited time to invest when they have so much going on with their profession. The only participant who reported they were neutral about the program stated that "it's not realistic when you are overworked and stressed" to try out some of the coping strategies. Teachers also mentioned that it was difficult to attend the in-person meeting outside of their typical workday, as many of them support extracurricular activities in the schools (e.g., coach athletic teams) and have their own childcare and family responsibilities. In contrast, there were a few teachers who actually reported they wished they had more in-person meetings as part of the program and opportunities to talk with other teachers more often, instead of an independent stress management program. 


\section{CHAPTER V: DISCUSSION}

The present study is important for furthering research and intervention efforts to improve teacher wellbeing and create a positive context for youth development in schools. Research has consistently demonstrated that teachers often experience high levels of stress (Kyriacou, 2001, 2011; Kyriacou \& Sutcliffe, 1978) and individual coping can be enhanced to mitigate the negative effects of this over time. The Stress Management for Teachers program is innovative in that is based on cognitive-behavioral science for coping, includes training in classroom management, and is relatively brief in comparison to existing programs (Herman \& Reinke, 2015). Overall the results from the study suggest that the program was effective on immediate outcomes of reducing stress and improving the use of adaptive coping strategies. There were medium treatment effects on teachers' mental health symptoms of anxiety and depression. While the program demonstrated effects for immediate and long-term individual outcomes of coping and mental health, there were no treatment effects on classroom competence or contextual factors in the classroom, perceptions of the school environment, and from observed interactions with students. The implications of study findings, comparisons to prior studies, connection with broader theories, limitations, areas for improvement, and recommendations for future studies are reviewed.

\section{Initial Program Efficacy}

Effects on Stress and Coping. From the multilevel model examining treatment effects, the intervention caused a reduction in perceptions of overall stress levels and this standardized effect size was medium $(d=0.64)$. The pre-post comparison from the teachers in the waitlist after they received the intervention also suggested a large effect (d 
$=-0.78)$ providing replication evidence that this program reduced teacher's perception of global stress. Interestingly, while the program had an effect on overall stress, there was a small but non-significant treatment effect $(d=-0.22)$ on the Teacher Stress Scale, which measures stressor specific to teachers including disruptive behaviors, managing paperwork, and physical symptoms of stress. This suggests that the program may have improved teacher's overall perception of stress but did not have an effect on specific stressors experienced in the classroom. It may be that the intervention was beneficial for improving teachers' perception of stress as a normal emotion and gave them strategies to cope with stressors, but did not sufficiently reduce the presence of stressors, such as workload demands or disruptive student behaviors.

The program also did not have an effect on the three components of burnout including emotional exhaustion, depersonalization, and personal accomplishment. Though the findings were not statistically significant, the standardized $b$ 's for emotional exhaustion were small (-0.40), and could suggest that the program has some treatment effect that would be detected in a larger sample. Replication with a larger sample size may be needed to clarify these findings. Conceptually, burnout is a longer-term experience that can develop as a result of working in a helping profession with prolonged stress and low coping over time (Maslach, 1991), so it may be that additional time is needed to see a change from improved coping impacting the experience of burnout, or additional individual or contextual supports could be needed to prevent this outcome.

Effects on Coping. The Stress Management for Teachers program specifically focused on improving coping behaviors and taught skills for inside and outside of the classroom. Promisingly, the intervention had effects on many of the proactive strategies 
introduced in the book and discussed in the supplemental trainings. The intervention had a small effect on active coping $(d=0.41)$ and planning $(d=0.66)$, which are strategies that are helpful for coping and preventing for ways to handle inevitable stressors. A preventative approach is beneficial for anticipating potential problems and having a greater sense of control to manage the response to them. There were also intervention effects on positive reframing, which is directly related to the adaptive thinking section from the stress management program. Cognitive restructuring is beneficial for altering negative thought patterns that can increase stress and anxiety (Beck \& Beck, 1995).

Interestingly, there was a small intervention effect on self-reported substance use including alcohol and other substances $(d=-0.34)$. The effect size in comparing pre-post with the waitlist condition was medium, though non-significant $(n=24, \mathrm{~d}=-0.53)$. The program did not directly address minimizing substance use as a coping strategy, but perhaps providing teachers with more adaptive strategies for coping reduced participant's use of substances to manage stress. Alternatively, it could be that this finding was influenced by social desirability since the participants in the intervention had increased interaction with the stress management coach, and following the intervention did not want to report substance use. This finding should be cautiously interpreted because it is based on the brief subscale from the longer measure and the practical significance of the meaning only suggests a 1-point reduction on a 4-point Likert scale. Despite the limitations of this findings, it provides an interesting consideration for future research into coping behaviors and substance use in the teaching profession.

Change in Coping over Time. The weekly rating forms also demonstrated the change in coping over time. From Bandura's (1977) theory of self-efficacy, cognitive 
beliefs about one's knowledge, understanding, and confidence to apply coping strategies are important for influencing actual behavior change and the use of new strategies. From examining the change over time, there were treatment effects on teachers' weekly perceptions of their coping as well as their confidence in their abilities to cope with stress. These findings provide support for the efficacy of the program in increasing teachers' coping and beliefs that they have the ability to manage their own stress. Essential to the success of the program, the intervention had an effect on the reported use of coping strategies. Not only did teachers learn strategies, but they increased their use of coping as a result of the program.

Another interesting finding is all of the teachers in the program, including the waitlist control teachers increased in their awareness and understanding of coping strategies through the course of the intervention. It may be that simply by agreeing to participate in the program and completing baseline and weekly rating forms of stress increased their awareness of their stress levels and ways to cope with stress. Selfreflection is a form of self-monitoring, which as an independent practice can cause positive changes (Harmon et al., 1980).

The findings from the before and after intervention comparisons for the waitlist teachers adds additional support for the findings that the program was beneficial in improving teachers' coping. In the sample of 24 teachers, there were significant improvements in self-reported awareness $(\mathrm{d}=1.20, p<.0001)$, knowledge $(\mathrm{d}=0.74, p$ $<.0001)$ and use $(\mathrm{d}=0.57, p=.001)$ of coping strategies after they received the intervention. Overall, results demonstrate that the stress management program increased 
teachers' perceptions of overall coping abilities and increased their confidence and use of adaptive coping strategies.

Mental Health. The effects of the program also extended to teachers' report of anxiety symptoms. These findings are compelling as they show that $50 \%$ of teachers in the intervention condition had improvements in anxiety severity and in contrast only $11.5 \%$ improved in the control group. Also in the control condition $23.1 \%$ of teachers increased in anxiety symptoms whereas only $3.8 \%$ of teachers in the intervention had an increase in clinical severity. The intervention effects on clinical symptom severity were statistically significant, and had a large effect size (Cramer's $\mathrm{V}=.42, p=.005)$.

While the regression modelling had non-significant effects for the intervention on the PHQ-8 measure $(p=.08)$, the effect size was small to medium $(d=0.48)$. In examining individual changes in depression severity, $42.3 \%$ of teachers in the intervention condition had improvements, while only $11.5 \%$ of teachers in the control condition improved. For the control condition $34.6 \%$ worsened in symptom severity while $7.7 \%$ of teachers had increased symptoms. From a chi-square analysis, these findings for changes in symptom severity level on the PHQ-8 were statistically significant (Cramer's $\mathrm{V}=.42, p=.01)$.

Decreases in mental health symptoms are beneficial for supporting emotional health and well-being of teachers. This is also important from an organizational perspective in creating a positive work climate by focusing on improving teachers' outcomes of mental health. Further, it's likely these mental health benefits are also related to student outcomes as well. When teachers have reduced mental health symptoms, this is associated with improved instructional quality and socio-emotional 
learning (McLean, et al., 2018; McLean \& Connor, 2015). The intervention effects on anxiety and potentially depression suggest that the program meaningfully influenced long-term and persistent outcomes related to mental health.

Null Findings. The intervention did not have treatment effects on some aspects of individual outcomes of stress specifically teacher-specific stressors and burnout, as previously discussed. The program also did not have any treatment effects on perceptions of organizational health, job satisfaction, or on teachers' sense of efficacy or effective classroom management behaviors in the classroom from the observations. While classroom management was included in the intervention, perhaps a stronger emphasis on this component would be needed to increase teachers' confidence in classroom management. A webinar may not have been a sufficient dose to impact efficacy and use of new behavioral strategies in the classroom. Prior studies in classroom management suggest that a supportive coaching model can be especially helpful for teachers to make positive classroom changes (Reinke, Stormont, Herman, \& Newcomer, 2013).

Connection to Theory. The Coping-Competence-Context Model of Teacher Stress (3C Model) reviewed in the introduction is a relevant theory to conceptualize these study findings (Herman et al., 2020). The program directly addressed the individual coping and mindset pathway from this theory through all modes of intervention delivery with the bibliotherapy, in-person training, and webinars. The results support the theory that individual coping can be enhanced, which can then reduce teacher stress and affect more distal outcomes of teacher mental health and well-being. The competence pathway from the $3 \mathrm{C}$ model for improving classroom management was also included as a smaller component of one of the online webinar trainings. As previously discussed, the 
intervention did not have treatment effects on observed classroom behaviors or teachers'

reported efficacy. Enhancing this aspect of the intervention may be needed, especially if individuals struggle with this aspect of teaching. The contextual pathway of broader systemic factors in the school and district influencing teacher stressors was not directly addressed through the intervention, so it may be understandable that there were no treatment effects on perceptions of occupational health. Overall the findings show empirical support for the pathways identified in the $3 \mathrm{C}$ model. The model also suggests possible areas for intervention improvement by addressing teachers' competence and improving contextual factors that influence teacher stress.

\section{Comparison to other Teacher Stress Programs.}

Intervention Effects. In comparing the results of this program to previous stress management interventions for teachers, the findings were similar. Of the mindfulness programs previously reviewed, the SMART program evaluated by Roeser and colleagues (2013) demonstrated the strongest intervention effects on occupational stress $(d=-0.57$ and -0.73 at 3 -month follow up), burnout ( $d=-0.76$ and -0.68 at 3 -month follow up), depression ( $d=-1.06$ and -1.56 at 3 -month follow up), anxiety $(d=-0.71$ and -1.01 at $3-$ month follow up). The CARE program evaluated through the largest randomized control trial of teacher stress programs with 226 teachers had smaller effects in intervention studies and did not have statistically significant treatment effects on anxiety or depression. The effects of the program seem to be similar or greater than treatment effects on teacher stress and mental health symptoms. The CARE program did demonstrate treatment effects on the classroom observations $(d=0.23)$ on the positive 
component of the CLASS rating scale; however, this current study is be underpowered to detect the small treatment effect on classroom observations.

Program Feasibility. Since teachers often report having limited time to accomplish all of the occupational demands (Kyriacou, 2001), the time investment of the intervention is critical to consider in comparing program effects. Overall, the current program required a far smaller time and resource investment for teachers and schools in comparison to other programs. This current program involved only one in-person session which lasted 1.5 hours, and then an additional maximum of 1.5 hours of independent videos, and reading from the book that teachers could fit into their own schedule over the course of three weeks. In contrast, the SMART program involved 11 sessions with 36 hours of in-person contact and 15 minutes a day of required journaling over the course of 8 weeks (Roeser et al., 2013). The CARE program was similarly time and resource intensive requiring 5 professional development days with 30 hours of in-person training. At an additional cost, teachers in the CARE program were compensated for their participation at their hourly rate and substitute's teachers were hired to cover the days missed for the training (Jennings et al., 2017). Prior cognitive-behavioral programs also involved a greater time commitment with the program by Forman (1982) including six 3hour sessions on Saturday mornings. The shortest stress management program examined in a randomized control trial with teachers was based off of the German AGIL training (Lehr et al., 2007) that involved two 8-hour days of training and a 3-hour follow-up training (Dicke et al., 2015). In summary, this program required $87 \%$ less time for an inperson meeting compared to the shortest stress management program, and $95 \%$ less times 
in comparison to the longest program. It's critical to consider this large difference in time commitment when interpreting the treatment effects.

Even though this program was the by far the least time intensive of the reviewed stress management programs, the most frequently reported barrier to this program from the qualitative feedback on the program by $29 \%$ of teachers was not having enough time to dedicate to the program. This highlights the importance of creating short-term effective interventions that are going to reach teachers who may already be feeling overwhelmed by the number of daily tasks and occupational responsibilities.

\section{Limitations}

There are important limitations that warrant consideration in interpreting the findings of the efficacy study of the Stress Management for Teachers program.

\section{Recruitment}

The first challenge of the study was recruiting participants. Initially all of the teachers in a large local district were emailed about the opportunity to participate, and yet few elected to take part. Additional efforts were needed to advertise and recruit teachers to take part in the program. Even still, many teachers who initially expressed interest in taking part in the study did not follow up on contact to participate. The initial proposed sample was 100 teachers, and 52 teachers participated following two phases of recruitment. One district provided additional incentive for teachers to participate by offering professional development credit. Anecdotally, the most common reason for not participating was not having enough time. Since the job is inherently demanding, it was challenging for teachers to add an additional commitment, even if the program could be helpful long-term. This may have impacted the sampling in that perhaps teachers who 
were really struggling took part, and this may be suggested by the averages of anxiety and depression for the teachers in the intervention were in moderate ranges. The challenge with recruitment influences the generalizability of the findings, as there may be differences in the teachers who elected to participate compared those who did not. In the future, evaluating the program as a school-wide professional development training could inform the effects of the intervention on a representative sample of teachers.

\section{Fidelity to Intervention}

The second limitation of the study was the variability in the use of intervention components and insufficient tracking fidelity of which teachers watched which webinars. This information would have improved understanding of the treatment effects and is recommended to be included in future studies. Clarifying the use and the intervention components will important for future evaluations of this program.

Different doses of the intervention could have an impact on intervention effects. All of the teachers participated in the in-person training which reviewed the teacher coping model and introduced strategies for adaptive thinking. The lessons on adaptive behaviors, classroom management, and long-term wellness were all presented through the online format and in later chapters in the book. If participants had low fidelity during the independent section of the intervention, they may have missed important components, especially related to strategies for handling behaviors in the classroom. The fact that this core intervention part may have been missed may explain the null findings of the intervention effects on classroom behaviors of teachers and students. In the future, a better system of tracking participant use of the online components could be helpful to compare intervention effects based on fidelity to the treatment. Another potential change 
to the program could be another in-person meeting to discuss classroom management strategies.

\section{Clarification about Individual Coping}

Finally, an important conceptual clarification to the program is that by emphasizing individual coping, this could suggest that teachers are struggling with stress because they do not have effective coping strategies; however, this is not case. The high prevalence of stress and burnout (Kyriacou, 2006) do not suggest individual failings in coping abilities, but rather a systemic problem. Theories of teacher stress emphasize widespread sources of stress that influence teacher outcomes including low pay, high-stakes testing, administrative demands, and limited behavioral supports (von der Embse, Sandalos, Pendergast \& Mankin, 2016). Improving coping in schools may be beneficial for reducing the intensity of stress enough for teachers to get by, but until the working conditions are improved coping skills can only go so far in improving teacher and student outcomes.

\section{Long-term Effects}

A final limitation to the current study is that the follow-up only looked at one time point to measure intervention effects four to six weeks later, but did not examine if the effects persisted over time. Future studies could examine to extent to which the treatment effects last, and if additional supports are needed over time to sustain positive results of the program over time.

\section{Future Directions}

Despite the limitations, this initial randomized control trial had many strengths with the randomized control design, improvements on prior study methodologies, and the 
feasibility of the intervention in comparison to other existing programs. The Stress Management for Teachers program demonstrated evidence for initial efficacy in increasing coping, reducing stress and mental health symptoms. This study suggests many areas for future research and refinements to the intervention to best support teachers and the students they teach.

\section{Classroom Management}

The first area for future refinement of the intervention implementation is creating a system that not only supports individual teachers' coping and perceptions of stress, but also addresses teaching efficacy and competency, especially in regards to classroom management (Herman et al., 2020). Additional emphasis on classroom management in the program through another in-person training specifically focused on behavioral strategies may be indicated for some teachers who perceive disruptive student behavior as a main source of stress. Especially for teachers new in the profession, prior studies have found similar treatment effects on stress from programs solely focused on classroom management in comparison to stress management programs (Dicke et al., 2015, Sharp \& Forman, 1985). A potential future study could be to replicate the design of these studies and compare the efficacy of stress management only intervention to an intervention involving stress management and classroom management training combined to see if the combined program improves competency and reduces classroom stressors.

\section{Contextual Supports}

School climate and contextual factors influencing stress for teachers is another critical area for intervention (Herman et al., 2020). Without support from school leadership and changes from an administrative level, supporting teachers with coping 
skills is limited. In a way, relying on individual coping skills training may send an unhelpful message that only teachers are responsible for improving their working conditions. If many teachers in a school are struggling to manage occupational demands, then perhaps addresses common systemic source of stress may more efficiently cause positive changes. Reducing workload and talking to teachers' about school-level changes that can improve the context is likely to be more helpful. How and why the program is delivered to teachers within a school system is essential to its success. Sufficient compensation, well-designed efficient professional development, and a positive working environment that values well-being is essential to improving teacher coping and the school context. Adding an additional intervention training component for administrators on effective school leadership and organizational health is a potential area for further refinement of the program.

\section{Levels of Intervention}

An additional area for future research is determining how to effectively assess for teachers' needs and implement this stress management program within a school system in an efficient and cost-effective way. This is challenging given the varying stressors and needs of individual teachers, and the limited time and resources in many school systems. Not all teachers need a highly intensive intervention, and many teachers may be effectively coping with the stress of their jobs (Herman et al., 2018). While limited time was the most frequently reported barrier to the stress management program, there were a few teachers (11\%) who reported they would have appreciated having more in-person contact and additional meetings. 
Just as interventions for students can be conceptualized from a three-tiered level of intervention, teachers' need for support can examined through this lens. Many teachers may benefit from a preventative group training, like the in-person training that could easily be delivered as a school or district level professional development training. Some teachers may desire additional on-going support for coping with stress from school level providers such a school counselors, social workers, or school psychologists who have the skills to provide brief, solution-focused supports for coping. Then some teachers struggling with serious mental health symptoms of depression and anxiety may need ongoing therapy from community providers.

Another potential study or alternative method for intervention implementation could be using an adaptive approach based on the needs of teachers. Some teachers may need support for individual coping, or some teachers may need more support for immediate classroom stressors through a classroom management intervention, or some teachers may need greater administrative support. A Sequential Multiple Assignment Randomized Trail (SMART) design could be used to evaluate the efficacy of this type of treatment assignment to condition based on the needs in order to provide the best tailored intervention for teachers (Murphy, 2005).

\section{Conclusion and Summary}

Teachers have incredible potential to positively influence many children throughout their careers. Enhancing teachers' wellbeing increases their abilities to create positive relationships with students, model social-emotional skills, and lead instruction in effective ways (Hamre \& Pianta, Jennings \& Greenberg, 2010; Roorda et al., 2011). Providing teachers with effective and useful coping strategies to reduce the high stress of 
their jobs is beneficial for teachers, students, and the broader educational context. Existing programs show that coping can be improved; however, many of these programs are time-intensive and recently have focused primarily on mindfulness training which not be useful for all teachers. This study examined the Stress Management for Teachers program that reduces the time and cost of previous programs by only involving a bibliotherapy, one in-person meeting, and online webinars. From this initial efficacy study, the intervention had positive effects on proximal outcomes of stress and coping behaviors, as well as distal outcomes related to mental health. The intervention did not have effects on long-term outcomes of job satisfaction, efficacy, organizational health, and downstream effects on observed behaviors. The results suggest that the intervention was beneficial for individual outcomes and the effects of this intervention are comparable and even greater than similar evaluation studies of stress management programs for teachers, involving a much more intensive time commitments for teachers. Additional refinements to reduce contextual stressors through administrative and systemic changes and additional supports for classroom management are critical areas for future research. 


\section{References}

Ahola, K., Pulkki-Råback, L., Kouvonen, A., Rossi, H., Aromaa, A., \& Lönnqvist, J. (2012). Burnout and behavior-related health risk factors: Results from the population-based Finnish Health 2000 study. Journal of Occupational and Environmental Medicine, 54(1), 17-22. doi:10.1097/JOM.0b013e31823ea9d9

Ainsworth, M. S. (1979). Infant-mother attachment. American Psychologist, 34(10), 932937. doi:10.1037/0003-066X.34.10.932

Arens, A. K., \& Morin, A. J. S. (2016). Relations between teachers' emotional exhaustion and students' educational outcomes. Journal of Educational Psychology, 108(6), 800-813. doi:10.1037/edu0000105

Barnes, G., Crowe, E., \& Schaefer, B. (2007). The cost of teacher turnover in five school districts: A pilot study. https://edsource.org/wp-content/uploads/old/NCTAF$\underline{\text { Cost-of-Teacher-Turnover-2007-full-report.pdf }}$

Beck, J. S., \& Beck, A. T. (1995). Cognitive therapy: Basics and beyond. New York: Guilford press.

Bellingrath, S., Weigl, T., \& Kudielka, B. M. (2009). Chronic work stress and exhaustion is associated with higher allostastic load in female school teachers. Stress: The International Journal on the Biology of Stress, 12(1), 37-48.

doi:10.1080/10253890802042041

Benjamini, Y., \& Hochberg, Y. (1995). Controlling the false discovery rate: a practical and powerful approach to multiple testing. Journal of the Royal statistical society: series B (Methodological), 57(1), 289-300. https://www.jstor.org/stable/2346101 
Benn, R., Akiva, T., Arel, S., \& Roeser, R. W. (2012). Mindfulness training effects for parents and educators of children with special needs. Developmental Psychology, 48(5), 1476-1487. doi:10.1037/a0027537

Bowlby, J. (1969). Attachment and loss v. 3 (Vol. 1). Random House. Furman, W., \& Buhrmester, D.(2009). Methods and measures: The network of relationships inventory: Behavioral systems version. International Journal of Behavioral Development, 33, 470-478.

Bowlby, J. (1988). A secure base: Parent-child attachment and healthy human development. New York, NY, US: Basic Books.

Boyle, G. J., Borg, M. G., Falzon, J. M., \& Baglioni, A. J. (1995). A structural model of the dimensions of teacher stress. British Journal of Educational Psychology, 65(1), 49-67. doi:doi:10.1111/j.2044-8279.1995.tb01130.x

Bretherton, I. (1985). Attachment theory: Retrospect and prospect. Monographs of the Society for Research in Child Development, 50(1-2), 3-35. doi:10.2307/3333824

Bronfenbrenner, U. (1994). Ecological models of human development. In International Encyclopedia of Education (2 ed., Vol. 3, pp. 37-43). Oxford: Elsevier.

Bronfenbrenner, U., \& Ceci, S. J. (1994). Nature-nuture reconceptualized in developmental perspective: A bioecological model. Psychological review, 101(4), 568-586. doi:10.1037/0033-295X.101.4.568

Bronfenbrenner, U., \& Morris, P. A. (2006). The Bioecological Model of Human Development. In Handbook of child psychology: Theoretical models of human development, Vol. 1, 6th ed. (pp. 793-828). Hoboken, NJ, US: John Wiley \& Sons Inc. 
Buyse, E., Verschueren, K., \& Doumen, S. (2011). Preschoolers' attachment to mother and risk for adjustment problems in Kindergarten: Can teachers make a difference? Social Development, 20(1), 33-50. doi:doi:10.1111/j.14679507.2009.00555.x

Byrne, B. M. (1993). The Maslach Burnout Inventory: Testing for factorial validity and invariance across elementary, intermediate and secondary teachers. Journal of Occupational and Organizational Psychology, 66(3), 197-212. doi:10.1111/j.2044-8325.1993.tb00532.x

Carver, C. S. (1997). You want to measure coping but your protocol's too long: Consider the Brief COPE. International Journal of Behavioral Medicine, 4(1), 92-100. doi:10.1207/s15327558ijbm0401_6

Carver, C. S., Scheier, M. F., \& Weintraub, J. K. (1989). Assessing coping strategies: A theoretically based approach. Journal of Personality and Social Psychology, 56(2), 267-283. doi:10.1037/0022-3514.56.2.267

Cecil, M. A., \& Forman, S. G. (1990). Effects of stress inoculation training and coworker support groups on teachers' stress. Journal of School Psychology, 28(2), 105-118. doi:10.1016/0022-4405(90)90002-O

Chrousos, G. P. (2009). Stress and disorders of the stress system. Nature Reviews Endocrinology, 5, 374. doi:10.1038/nrendo.2009.106

Cohen, J. (1988). Statistical power analysis for the behavioral sciences (2nd ed.): Erlbaum Associates, Hillsdale.

Cohen, S., Kamarck, T., \& Mermelstein, R. (1983). A global measure of perceived stress. Journal of Health and Social Behavior, 24(4), 385-396. doi:10.2307/2136404 
Cropanzano, R., Rupp, D. E., \& Byrne, Z. S. (2003). The relationship of emotional exhaustion to work attitudes, job performance, and organizational citizenship behaviors. Journal of Applied Psychology, 88(1), 160-169. doi:10.1037/00219010.88 .1 .160

Cronbach, L. J. (1951). Coefficient alpha and the internal structure of tests. Psychometrika, 16, 297-334. http://dx.doi.org/10.1007/BF02310555

Cuijpers, P. (1998). A psychoeducational approach to the treatment of deppression. Behavior Therapy, 29, 3, 521-533. doi: https //doi.org/10.1016/S0005 7894(98)80047 6 .

Cullen, M., \& Wallace, L. (2010). Stress management and relaxation techniques in education (SMART) training manual. Aurora, Colorado: Impact Foundation.

Darr, W., \& Johns, G. (2008). Work strain, health, and absenteeism: A meta-analysis. Journal of occupational health psychology, 13(4), 293-318. doi:10.1037/a0012639

Davis, H. A. (2003). Conceptualizing the role and influence of student-teacher relationships on children's social and cognitive development. Educational psychologist, 38(4), 207-234. doi:10.1207/S15326985EP3804_2

Dicke, T., Elling, J., Schmeck, A., \& Leutner, D. (2015). Reducing reality shock: The effects of classroom management skills training on beginning teachers. Teaching and Teacher Education, 48, 1-12. doi:10.1016/j.tate.2015.01.013

Dong, N., \& Maynard, R. A. (2013). PowerUp!: A tool for calculating minimum detectable effect sizes and sample size requirements for experimental and quasi- 
experimental designs. Journal of Research on Educational Effectiveness, 6(1), 2467. doi:10.1080/19345747.2012.673143

Duffy, R. D., \& Lent, R. W. (2009). Test of a social cognitive model of work satisfaction in teachers. Journal of Vocational Behavior, 75(2), 212-223. doi:10.1016/j.jvb.2009.06.001

Eddy, C. L., Herman, K. C., \& Reinke, W. M. (2017). Single-item teacher stress and coping measures. Paper presented at the Annual American Psychological Association Conference, Washington D.C.

Ellis, A. (1991). The revised ABC's of rational-emotive therapy (RET). Journal of Rational-Emotive \& Cognitive-Behavior Therapy, 9(3), 139-172. doi:10.1007/BF01061227

Fimian, M. J. (1984). The development of an instrument to measure occupational stress in teachers: The Teacher Stress Inventory. Journal of Occupational Psychology, 57(4), 277-293. doi:doi:10.1111/j.2044-8325.1984.tb00169.x

Fimian, M. J., \& Fastenau, P. S. (1990). The validity and reliability of the Teacher Stress Inventory: A re-analysis of aggregate data. Journal of Organizational Behavior, 11(2), 151-157. doi:10.1002/job.4030110206

Forman, S. G. (1982). Stress management for teachers: A cognitive-behavioral program. Journal of School Psychology, 20(3), 180-187. doi:10.1016/0022-4405(82)900474

Gottfredson, D. C., Cook, T. D., Gardner, F. E. M., Gorman-Smith, D., Howe, G. W., Sandler, I. N., \& Zafft, K. M. (2015). Standards of evidence for efficacy, 
effectiveness, and scale-up research in prevention science: Next generation. Journal of Prevention Science, 16(7), 893-926. doi:10.1007/s11121-015-0555-x

Gregory, A., \& Ripski, M. B. (2008). Adolescent trust in teachers: Implications for behavior in the high school classroom. School Psychology Review, 37(3), 337353.

Griffith, J., Steptoe, A., \& Cropley, M. (1999). An investigation of coping strategies associated with job stress in teachers. British Journal of Educational Psychology, 69(4), 517-531. doi:10.1348/000709999157879

Grossman, P., Niemann, L., Schmidt, S., \& Walach, H. (2004). Mindfulness-based stress reduction and health benefits: A meta-analysis. Journal of Psychosomatic Research, 57(1), 35-43. doi:https://doi.org/10.1016/S0022-3999(03)00573-7

Hamre, B. K., \& Pianta, R. C. (2001). Early teacher-child relationships and the trajectory of children's school outcomes through eighth grade. Child Development, 72(2), 625-638. doi:10.1111/1467-8624.00301

Harmon, T. M., Nelson, R. O., \& Hayes, C. (1980). Self-Monitoring of mood versus activity by depressed clients. Journal of Consulting and Clinical Psychology, 48(1), 30-38. https://doi.org/10.1037/0022-006X.48.1.30

Herman, K. C., Hickmon-Rosa, J., \& Reinke, W. M. (2018). Empirically derived profiles of teacher stress, burnout, self-efficacy, and coping and associated student outcomes. Journal of Positive Behavior Interventions, 20(2), 90-100. doi:10.1177/1098300717732066

Herman, K. C., \& Reinke, W. M. (2012). Stress and Coping Items. Columbia, MO: University of Missouri. 
Herman, K. C., \& Reinke, W. M. (2015). Stress management training for teachers: A proactive guide. New York, NY: Guilford.

Ho, C.-L., \& Au, W.-T. (2006). Teaching Satisfaction Scale:Measuring Job Satisfaction of Teachers. Educational and Psychological Measurement, 66(1), 172-185. doi: $10.1177 / 0013164405278573$

Hofmann, S. G., Asnaani, A., Vonk, I. J. J., Sawyer, A. T., \& Fang, A. (2012). The efficacy of Cognitive Behavioral Therapy: A review of meta-analyses. Cognitive therapy and research, 36(5), 427-440. doi:10.1007/s10608-012-9476-1

Hoy, W. K., \& Fedman, J. A. (1987). Organizational health: The concept and its measure. Journal of research and Development in Education, 20(4), 30-37.

Ingersoll, R. M. (2001). Teacher turnover and teacher shortages: An organizational analysis. American educational research journal, 38(3), 499-534. doi:10.3102/00028312038003499

Ingersoll, R. M., \& Smith, T. M. (2003). The wrong solution to the teacher shortage. Jackson, S. E., \& Maslach, C. (1982). After-effects of job-related stress: Families as victims. Journal of Organizational Behavior, 3(1), 63-77. doi:doi:10.1002/job.4030030106

Jeffcoat, T., \& Hayes, S. C. (2012). A randomized trial of ACT bibliotherapy on the mental health of K-12 teachers and staff. Behaviour Research and Therapy, 50(9), $571-579$.

Jennings, P. A., Brown, J. L., Frank, J. L., Doyle, S., Oh, Y., Davis, R., . . Greenberg, M. T. (2017). Impacts of the CARE for teachers program on teachers' social and 
emotional competence and classroom interactions. Journal of Educational Psychology, 109(7), 1010-1028. doi:10.1037/edu0000187

Jennings, P. A., \& DeMauro, A. A. (2017). Individual-level interventions: Mindfulnessbased approaches to reducing stress and improving performance among teachers. In T. M. McIntyre, S. E. McIntyre, \& D. J. Francis (Eds.), Educator Stress: An Occupational Health Perspective (pp. 319-346). Cham, Switzerland: Springer International Publishing.

Jennings, P. A., Frank, J. L., Snowberg, K. E., Coccia, M. A., \& Greenberg, M. T. (2013). Improving classroom learning environments by Cultivating Awareness and Resilience in Education (CARE): Results of a randomized controlled trial. School Psychology Quarterly, 28(4), 374-390. doi:10.1037/spq0000035

Jennings, P. A., \& Greenberg, M. T. (2009). The prosocial classroom: Teacher social and emotional competence in relation to student and classroom outcomes. Review of Educational Research, 79(1), 491-525. doi:10.3102/0034654308325693

Johnson, S., Cooper, C., Cartwright, S., Donald, I., Taylor, P., \& Millet, C. (2005). The experience of work-related stress across occupations. Journal of Managerial Psychology, 20(2), 178-187. doi:10.1108/02683940510579803

Kabat-Zinn, J. (2003). Mindfulness-based interventions in context: Past, present, and future. Clinical Psychology: Science and Practice, 10(2), 144-156. doi:10.1093/clipsy/bpg016

Kelley, K. (2007). Methods for the Behavioral, Educational, and Social Sciences: An R Package. Behavior Research Methods, 39, 979-984. 
Kilfedder, C., Power, K., Karatzias, T., McCafferty, A. Niven, K., Chouliara, Z., Galloway, L. \& Sharp, S. (2010). A randomized trial of face-to-face counselling versus telephone counseling versus bibliotherapy for occupational stress. Psychology and Psychotherapy: theory, research, and practice, 83, 223-242.

Kim, J., Shin, Y., Tsukayama, E., \& Park, D. (2020). Stress mindset predicts job turnover among preschool teachers. Journal of School Psychology, 78, 13-22. https://doi.org/10.1016/j.jsp.2019.11.002

Klassen, R. M., Bong, M., Usher, E. L., Chong, W. H., Huan, V. S., Wong, I. Y. F., \& Georgiou, T. (2009). Exploring the validity of a teachers' self-efficacy scale in five countries. Contemporary Educational Psychology, 34(1), 67-76. doi:10.1016/j.cedpsych.2008.08.001

Klusmann, U., Kunter, M., Trautwein, U., Lüdtke, O., \& Baumert, J. (2008). Engagement and emotional exhaustion in teachers: Does the school context make a difference? Applied Psychology, 57, 127-151. doi:10.1111/j.1464-0597.2008.00358.x

Kokkinos, C. M. (2007). Job stressors, personality and burnout in primary school teachers. British Journal of Educational Psychology, 77(1), 229-243. doi:10.1348/000709905X90344

Kroenke, K., Strine, T. W., Spitzer, R. L., Williams, J. B. W., Berry, J. T., \& Mokdad, A. H. (2009). The PHQ-8 as a measure of current depression in the general population. Journal of Affective Disorders, 114(1-3), 163-173. doi:10.1016/j.jad.2008.06.026

Kyriacou, C. (2001). Teacher stress: Directions for future research. Educational Review, 53(1), 27-35. doi:0.1080/0013191012003362 
Kyriacou, C. (2011). Teacher stress: From prevalence to resilience. In Handbook of stress in the occupations. (pp. 161-173). Northampton, MA, US: Edward Elgar Publishing.

Kyriacou, C., \& Sutcliffe, J. (1978). A model of teacher stress. Educational studies, 4(1), 1-6. doi:10.1080/0305569780040101

Lazarus, R. S., \& Folkman, S. (1984). Coping and adaptation. The handbook of behavioral medicine, 282-325.

Lazarus, R. S., \& Folkman, S. (1987). Transactional theory and research on emotions and coping. European Journal of Personality, 1(3, Spec Issue), 141-169. doi:doi:10.1002/per.2410010304

Maslach, C., Jackson, S. E., \& Leiter, M. P. (1986). Maslach Burnout Inventory. Palo Alto. In: CA: Consulting psychologists press.

Maslach, C., Schaufeli, W. B., \& Leiter, M. P. (2001). Job burnout. Annual review of psychology, 52(1), 397-422. doi:10.1146/annurev.psych.52.1.397

Masten, A. S., Roisman, G. I., Long, J. D., Burt, K. B., Obradović, J., Riley, J. R., . . . Tellegen, A. (2005). Developmental cascades: Linking academic achievement and externalizing and internalizing symptoms over 20 years. Developmental Psychology, 41(5), 733-746. doi:10.1037/0012-1649.41.5.733

McLean, L., \& Connor, C. M. (2015). Depressive symptoms in third-grade teachers: Relations to classroom quality and student achievement. Child Development, 86(3), 945-954. doi:10.1111/cdev.12344

McLean, L., Abry, T., Taylor, M., \& Connor, C. M. (2018). Associations among teachers' depressive symptoms and students' classroom instructional experiences in third 
grade. Journal of school psychology, 69, 154-168.

https://doi.org/10.1016/j.jsp.2018.05.002

Meichenbaum, D. (1977). Cognitive-Behavior modification: An integrative approach. New York, NY: Plenum Press.

Melamed, S., Shirom, A., Toker, S., Berliner, S., \& Shapira, I. (2006). Burnout and risk of cardiovascular disease: Evidence, possible causal paths, and promising research directions. Psychological Bulletin, 132(3), 327-353. doi:10.1037/00332909.132.3.327

Montgomery, C. (2017). Development and testing of a theoretical-empirical model of educator stress, coping and burnout. In T. M. McIntyre, S. E. McIntyre, \& D. J. Francis (Eds.), Educator Stress: An Occupational Health Perspective (pp. 181195). Cham: Springer International Publishing.

Montgomery, C., \& Rupp, A. A. (2005). A meta-analysis for exploring the diverse causes and effects of stress in teachers. Canadian Journal of Education/Revue canadienne de l'éducation, 458-486. doi:10.2307/4126479

Murphy, S. A. (2005). An experimental design for the development of adaptive treatment strategies. Statistics in medicine, 24(10), 1455-1481. https://doi.org/10.1002/sim.2022

National Center for Education Statistics Institute of Education Sciences. (2018). Back to school statistics. Retrieved from https://nces.ed.gov/fastfacts/display.asp?id=372

Act of 2001, 1425, Pub. L. No. Pub. L. No. 107-110, § 115 107-110 (2001).

Oberle, E., \& Schonert-Reichl, K. A. (2016). Stress contagion in the classroom? The link between classroom teacher burnout and morning cortisol in elementary school 
students. Social Science \& Medicine, 159, 30-37.

doi:10.1016/j.socscimed.2016.04.031

Pianta, R. C., Hamre, B., \& Stuhlman, M. (2003). Relationships between teachers and children. In Handbook of psychology: Educational psychology, Vol. 7. (pp. 199234). Hoboken, NJ, US: John Wiley \& Sons Inc.

Pianta, R. C., \& Steinberg, M. (1992). Teacher-child relationships and the process of adjusting to school. In Beyond the parent: The role of other adults in children's lives. (pp. 61-80). San Francisco, CA, US: Jossey-Bass.

Reinke, W. M., Herman, K. C., \& Stormont, M. (2013). Classroom-level Positive Behavior Supports in Schools implementing SW-PBIS:Identifying areas for enhancement. 15(1), 39-50. doi:10.1177/1098300712459079

Reinke, W. M., \& Newcomer, L. (2010). Brief classroom interaction observation revised (BCIO-R). Columbia, MO: University of Missouri.

Richardson, K. M., \& Rothstein, H. R. (2008). Effects of occupational stress management intervention programs: A meta-analysis. Journal of occupational health psychology, 13(1), 69-93. doi:10.1037/1076-8998.13.1.69

Rimm-Kaufman, S. E., \& Pianta, R. C. (2000). An ecological perspective on the transition to Kindergarten: A theoretical framework to guide empirical research. Journal of Applied Developmental Psychology, 21(5), 491-511. doi:https://doi.org/10.1016/S0193-3973(00)00051-4

Roeser, R. W., Schonert-Reichl, K. A., Jha, A., Cullen, M., Wallace, L., Wilensky, R., . . Harrison, J. (2013). Mindfulness training and reductions in teacher stress 
and burnout: Results from two randomized, waitlist-control field trials. Journal of Educational Psychology, 105(3), 787-804. doi:10.1037/a0032093

Ronfeldt, M., Loeb, S., \& Wyckoff, J. (2013). How teacher turnover harms student achievement. American educational research journal, 50(1), 4-36. doi:10.3102/0002831212463813

Roorda, D. L., Koomen, H. M. Y., Spilt, J. L., \& Oort, F. J. (2011). The influence of affective teacher-student relationships on students' school engagement and achievement:a meta-analytic approach. Review of Educational Research, 81(4), 493-529. doi:10.3102/0034654311421793

Rudasill, K. M., Reio, T. G., Stipanovic, N., \& Taylor, J. E. (2010). A longitudinal study of student-teacher relationship quality, difficult temperament, and risky behavior from childhood to early adolescence. Journal of School Psychology, 48(5), 389412. doi:https://doi.org/10.1016/j.jsp.2010.05.001

Ryan, S. V., von der Embse, N. P., Pendergast, L. L., Saeki, E., Segool, N., \& Schwing, S. (2017). Leaving the teaching profession: The role of teacher stress and educational accountability policies on turnover intent. Teaching and Teacher Education, 66, 1-11. doi:https://doi.org/10.1016/j.tate.2017.03.016

Sharma, M., \& Rush, S. E. (2014). Mindfulness-based stress reduction as a stress management intervention for healthy individuals:A systematic review. Journal of Evidence-Based Complementary \& Alternative Medicine, 19(4), 271-286. doi: $10.1177 / 2156587214543143$ 
Sharp, J. J., \& Forman, S. G. (1985). A comparison of two approaches to anxiety management for teachers. Behavior Therapy, 16(4), 370-383. doi:10.1016/s00057894(85)80004-6

Shin, H., Noh, H., Jang, Y., Park, Y. M., \& Lee, S. M. (2013). A longitudinal examination of the relationship between teacher burnout and depression. Journal of Employment Counseling, 50(3), 124-137. doi:10.1002/j.21611920.2013.00031.x

Simon, N. S., \& Johnson, S. M. (2015). Teacher turnover in high-poverty schools: What we know and can do. Teachers College Record, 117(3), 1-36.

Skaalvik, E. M., \& Skaalvik, S. (2011). Teacher job satisfaction and motivation to leave the teaching profession: Relations with school context, feeling of belonging, and emotional exhaustion. Teaching and Teacher Education, 27(6), 1029-1038. doi:https://doi.org/10.1016/j.tate.2011.04.001

Spitzer, R. L., Kroenke, K., Williams, J. B. W., \& Löwe, B. (2006). A brief measure for assessing generalized anxiety disorder: The GAD-7. Archives of Internal Medicine, 166(10), 1092-1097. doi:10.1001/archinte.166.10.1092

Steinhardt, M. A., Smith Jaggars, S. E., Faulk, K. E., \& Gloria, C. T. (2011). Chronic work stress and depressive symptoms: Assessing the mediating role of teacher burnout. Stress and Health, 27(5), 420-429. doi:doi:10.1002/smi.1394

Tapp, J. (2004). MOOSES (Multi-Option Observation System for Experimental Studies). Retrieved from http://kc.vanderbilt .edu/mooses/mooses.html 
Tschannen-Moran, M., \& Hoy, A. W. (2001). Teacher efficacy: Capturing an elusive construct. Teaching and Teacher Education, 17(7), 783-805. doi:10.1016/S0742051X(01)00036-1

Tudge, J. R. H., Mokrova, I., Hatfield, B. E., \& Karnik, R. B. (2009). Uses and misuses of Bronfenbrenner's Bioecological Theory of Human Development. Journal of Family Theory \& Review, 1(4), 198-210. doi:doi:10.1111/j.17562589.2009.00026.x

Turner, C., Lombardo, C., \& Logan, E. B. (2018). Teacher walkouts: A state-by-state guide. NPR. Retrieved from https://www.npr.org/sections/ed/2018/04/25/602859780/teacher-walkouts-a-stateby-state-guide

U. S. Bureau of Labor Statistics. (2018). Occupational Employment Statistics. Retrieved from https://www.bls.gov/oes/current/area_emp_chart/area_emp_chart.htm van der Klink, J. J. L., Blonk, R. W. B., Schene, A. H., \& van Dijk, F. J. H. (2001). The benefits of interventions for work-related stress. American Journal of Public Health, 91(2), 270-276. doi:10.2105/ajph.91.2.270

von der Embse, N., Ryan, S. V., Gibbs, T., \& Mankin, A. (2019). Teacher stress interventions: A systematic review. Psychology in the Schools, 56(8), 1328-1343. https://doi.org/10.1002/pits.22279

Watlington, E., Shockley, R., Guglielmino, P., \& Felsher, R. (2010). The high cost of leaving: An analysis of the cost of teacher turnover. Journal of Education Finance, 36(1), 22-37. doi:10.1353/jef.0.0028 
Wickham H (2016). ggplot2: Elegant Graphics for Data Analysis. Springer-Verlag New York. ISBN 978-3-319-24277-4, https://ggplot2.tidyverse.org. 


\section{Appendices}

Appendix A. Project Timeline

Appendix B. Weekly Self Monitoring Form

Appendix C. Theory of Change for Stress Management Intervention

Appendix D. Intervention Components

Appendix E. Tables and Figures

Appendix F. Open-ended Feedback on the Program from Teachers 
Appendix A.

Project Timeline

\begin{tabular}{|c|c|c|c|c|c|c|}
\hline Week & $1-2$ & 3 & 4 & 5 & 6 & $7-8$ \\
\hline Task & $\begin{array}{l}\text { Baseline } \\
\text { data } \\
\text { collection } \\
\text { (teachers } \\
\text { were sent } \\
\text { Qualtrics } \\
\text { surveys } \\
\text { through } \\
\text { email and } \\
\text { had two } \\
\text { weeks to } \\
\text { complete } \\
\text { the surveys) }\end{array}$ & $\begin{array}{l}\text { Intervention } \\
\text { begins } \\
\text { ( } 90 \text { minute in } \\
\text { person } \\
\text { trainings } \\
\text { completed - } \\
\text { targeted at a } \\
\text { maximum of } \\
3 \text { sites) } \\
\text { Chapters } 1-4\end{array}$ & $\begin{array}{l}\text { Online } \\
\text { Lesson } \\
\text { Chapters 5-6 }\end{array}$ & $\begin{array}{l}\text { Online } \\
\text { Lesson } \\
\text { Chapters 7-8 }\end{array}$ & $\begin{array}{l}\text { Online } \\
\text { Lesson } \\
\text { Chapters 9-13 }\end{array}$ & $\begin{array}{l}\text { Follow up } \\
\text { data } \\
\text { collection } \\
\text { (teachers } \\
\text { were sent } \\
\text { Qualtrics } \\
\text { surveys } \\
\text { through email } \\
\text { and had two } \\
\text { weeks to } \\
\text { complete the } \\
\text { surveys) }\end{array}$ \\
\hline Measures & $\begin{array}{l}\text { Baseline } \\
\text { measures } \\
\text { Assessment } \\
1\end{array}$ & & Assessment 2 & Assessment 3 & Assessment 4 & $\begin{array}{l}\text { Follow up } \\
\text { measures } \\
\text { Assessment } 5\end{array}$ \\
\hline $\begin{array}{l}\text { Estimated } \\
\text { Time for } \\
\text { Participants }\end{array}$ & $\begin{array}{l}\text { All } \\
\text { participants: } \\
30-45 \\
\text { minutes to } \\
\text { complete } \\
\text { surveys }\end{array}$ & $\begin{array}{l}\text { Intervention: } \\
90 \text { minutes } \\
\text { Control: none }\end{array}$ & $\begin{array}{l}\text { Intervention: } \\
90 \text { minutes } \\
\text { (30 minute } \\
\text { lesson }+30 \\
\text { minutes to } \\
\text { read the } \\
\text { chapters }+30 \\
\text { minutes total } \\
\text { of daily } \\
\text { practice) } \\
\text { Control: } 5 \\
\text { minutes for } \\
\text { survey }\end{array}$ & $\begin{array}{l}\text { Intervention: } \\
90 \text { minutes } \\
\text { (30 minute } \\
\text { lesson }+30 \\
\text { minutes to } \\
\text { read the } \\
\text { chapters }+30 \\
\text { minutes total } \\
\text { of daily } \\
\text { practice) } \\
\text { Control: } 5 \\
\text { minutes for } \\
\text { survey }\end{array}$ & $\begin{array}{l}\text { Intervention: } \\
90 \text { minutes } \\
(30 \text { minute } \\
\text { lesson }+30 \\
\text { minutes to } \\
\text { read the } \\
\text { chapters }+30 \\
\text { minutes total } \\
\text { of daily } \\
\text { practice) } \\
\text { Control: } 5 \\
\text { minutes for } \\
\text { survey }\end{array}$ & $\begin{array}{l}30-45 \\
\text { minutes to } \\
\text { complete } \\
\text { surveys }\end{array}$ \\
\hline Compensation & $25 \$$ & $5 \$$ & $5 \$$ & $5 \$$ & $5 \$$ & $25 \$$ \\
\hline
\end{tabular}

Total possible compensation per teacher: $70 \$$ 


\section{Appendix B.}

\section{Weekly Self-Monitoring Form}

How stressful is your job?

How well have you been coping with the stress of your job right now?

Check off as many of the following things have you done this week to cope with stress:

- Rated your mood

- Wrote about thoughts and feelings

$\circ$ Increased positive thinking

- Decreased negative thinking

- ABCDE method

○ Wrote a thank you note

- Tried out new strategies in the classroom

- Increased praise to students

- Spent time with friends or loved ones

- Did something that you enjoy

- Did something active

- Healthy eating

- Addressed a conflict with another person

○ Mindfulness or yoga

Was there anything else that helped you handle the stress of your job this week? Please describe: (open-ended) 
Appendix C.

Theory of Change for Stress Management Intervention

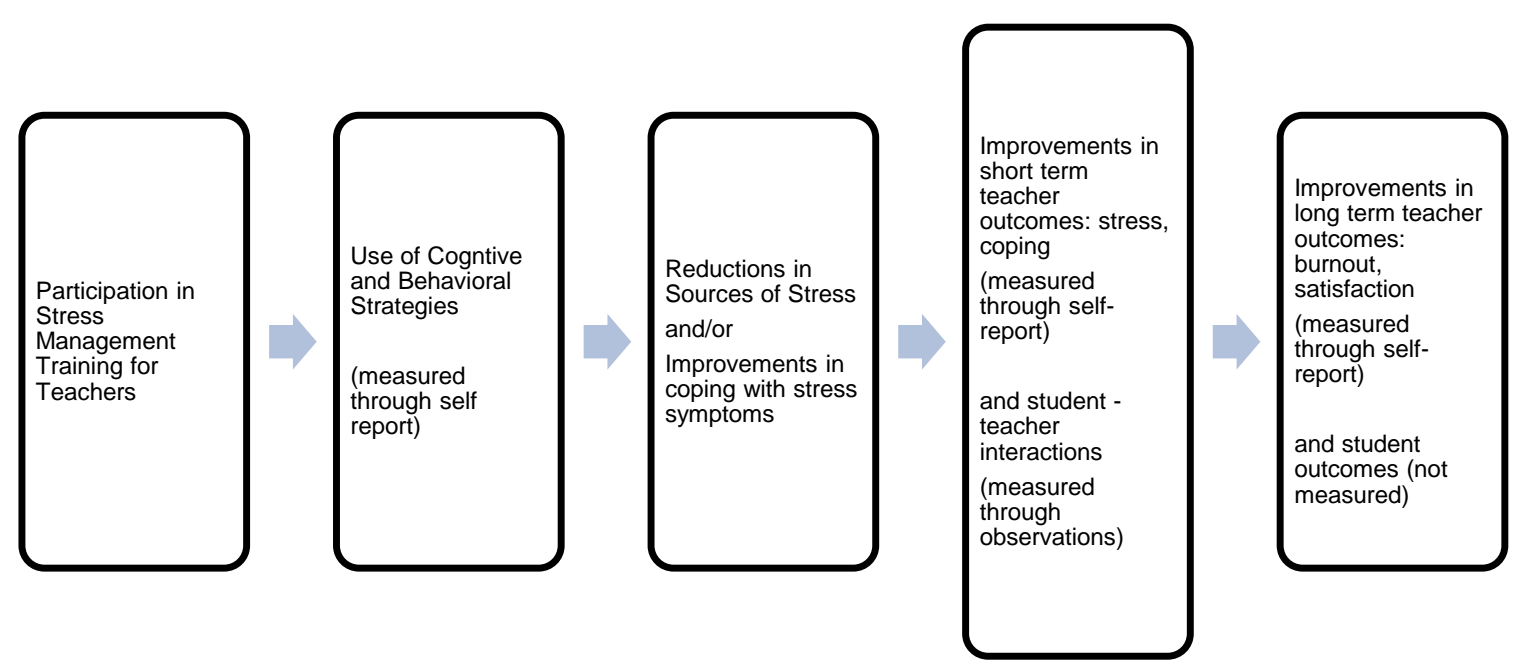




\section{Appendix D.}

\section{Intervention Components}

\section{OVERVIEW VIDEO}

This is about an hour and the first video of the program - https://youtu.be/wsxIHOKpf6Q After this video, there are three additional webinars that correspond with chapters in the book. It is suggested that teachers spend one week on each of the following lessons.

\section{ONLINE LESSON 1}

The first part of the webinar is to watch the overview video of the Adaptive Thinking chapters. After watching the overview video, pick at least one the remaining videos to learn about a specific strategy. The Positive/Negative thoughts method is from Chapter 5 in the book. The ABC Method is from Chapter 6 in the book.

Overview: https://www.youtube.com/watch?v=6FVNSMdBGas Positive/Negative Thoughts: https://www.youtube.com/watch?v=YaxMstIHG7U ABC Method: https://www.youtube.com/watch?v=sVltXLL650Q

\section{ONLINE LESSON 2}

The following videos have content from Chapters $7 \& 8$ from the book. The first two videos are about the Adaptive Behaviors chapter. You can learn about general adaptive behaviors or relaxation skills.

The third video is about increasing competence and self-efficacy, especially related to managing student behaviors. This comes from Chapter 8 from the book. You can also check out the Classroom Checkup website for more information: http://classroomcheckup.org/

Adaptive Behaviors: https://youtu.be/bFItkTNC730

Relaxation Strategies: https://youtu.be/h4VFaR10iWQ

Competence \& Self-efficacy: https://youtu.be/8VjSwfL5GU4

\section{ONLINE LESSON 3}

This is the third and final online lesson for the Stress Management program. Please watch this video with Dr. Keith Herman, one of the creators of the program, about creating a good personal development plan. If you're interested, also check out the video about Getting to Good/Beyond Survival. In this video I'll talk about the material from Chapter 9 about strategies for improving wellness beyond stress management.

Getting to Good: https://youtu.be/1tquf5VAj7w Long term planning/MI:https://youtu.be/eLO38bWkwOA 


\section{Appendix E.}

\section{Tables and Figures}

Table 1

Participant Demographic Information

\begin{tabular}{|c|c|c|c|c|c|c|}
\hline & \multicolumn{3}{|c|}{ Control $(n=27)$} & \multicolumn{3}{|c|}{ Treatment $(n=26)$} \\
\hline & $\%$ & Mean & SD & $\%$ & Mean & SD \\
\hline \multicolumn{7}{|l|}{ Gender } \\
\hline Female & 81 & & & 88 & & \\
\hline Male & 19 & & & 12 & & \\
\hline \multicolumn{7}{|l|}{ Race } \\
\hline White & 96 & & & 88 & & \\
\hline Black & 4 & & & 0 & & \\
\hline Asian & 0 & & & 8 & & \\
\hline Multiracial & 0 & & & 4 & & \\
\hline \multicolumn{7}{|l|}{ School Level } \\
\hline Elementary & 44 & & & 27 & & \\
\hline Middle & 30 & & & 23 & & \\
\hline High & 26 & & & 50 & & \\
\hline \multicolumn{7}{|l|}{ Education Status } \\
\hline General Education & 89 & & & 89 & & \\
\hline Special Education & 11 & & & 11 & & \\
\hline \multicolumn{7}{|l|}{ Highest Degree } \\
\hline Bachelor's & 56 & & & 42 & & \\
\hline Master's & 44 & & & 58 & & \\
\hline Age & & 39.44 & 12.21 & & 36.08 & 9.83 \\
\hline Years of teaching experience & & 10.67 & 9.33 & & 9.88 & 9.60 \\
\hline
\end{tabular}

Notes. ${ }^{1}$ Information for all of the teachers who enrolled in the study $(\mathrm{N}=53)$. 
Table 2

Study Measures

\begin{tabular}{|c|c|c|c|c|c|c|}
\hline & \multicolumn{3}{|c|}{ Pre Intervention } & \multicolumn{3}{|c|}{ Post Intervention } \\
\hline & Mean & SD & Omega & Mean & SD & Omega \\
\hline Perceived Stress Scale & 20.69 & 5.76 & .86 & 18.38 & 5.83 & .88 \\
\hline \multicolumn{7}{|l|}{ Maslach Burnout } \\
\hline Emotional Exhaustion & 39.60 & 11.76 & .91 & 38.85 & 11.38 & .91 \\
\hline Depersonalization & 13.69 & 6.26 & .89 & 13.57 & 5.99 & .78 \\
\hline Personal Accomplishment & 45.67 & 5.69 & .78 & 45.72 & 6.75 & .87 \\
\hline Teacher Stress Scale & 61.06 & 10.57 & .90 & 60.21 & 10.70 & .89 \\
\hline Stress & 7.94 & 1.49 & - & 7.53 & 1.96 & - \\
\hline Coping & 5.35 & 2.10 & - & 6.87 & 2.03 & - \\
\hline Confidence in Coping & 6.46 & 2.14 & - & 7.09 & 1.98 & - \\
\hline Awareness of Coping & 6.08 & 2.64 & - & 8.06 & 1.83 & - \\
\hline Understanding of Coping & 5.31 & 2.40 & - & 7.59 & 2.14 & - \\
\hline Use of Coping & 4.85 & 2.60 & - & 7.17 & 2.32 & - \\
\hline \multicolumn{7}{|l|}{ Brief Cope } \\
\hline Active Coping & 5.81 & 1.44 & .76 & 6.14 & 1.40 & .78 \\
\hline Humor & 4.71 & 2.08 & .88 & 4.69 & 1.75 & .84 \\
\hline Instrumental Support & 5.65 & 1.74 & .85 & 5.71 & 1.74 & .78 \\
\hline Planning & 5.94 & 1.47 & .73 & 6.23 & 1.23 & - \\
\hline Positive Reframing & 6.14 & 1.48 & .81 & 5.98 & 1.50 & .75 \\
\hline Use of Emotional Support & 5.52 & 1.72 & .85 & 5.75 & 1.40 & .83 \\
\hline Self-Blame & 5.31 & 1.67 & .67 & 4.62 & 1.91 & .84 \\
\hline Self-Distraction & 5.83 & 1.56 & .56 & 5.48 & 1.53 & .54 \\
\hline Substance Use & 1.46 & 0.75 & - & 1.56 & .87 & - \\
\hline Venting & 4.94 & 1.47 & .62 & 5.17 & 1.48 & .64 \\
\hline $\mathrm{GAD}-7$ & 10.81 & 5.36 & .89 & 9.72 & 5.29 & .91 \\
\hline PHQ - 8 & 9.87 & 5.81 & .87 & 8.72 & 6.03 & .90 \\
\hline Teaching Efficacy & 78.75 & 10.78 & .85 & 82.92 & 13.48 & .94 \\
\hline OHI - Collegial Leadership & 30.87 & 7.60 & .95 & 30.02 & 8.12 & .96 \\
\hline OHI - Teacher Affiliation & 26.08 & 4.74 & .89 & 25.96 & 4.53 & .88 \\
\hline Teacher Satisfaction & 14.48 & 3.13 & .87 & 14.60 & 3.31 & .88 \\
\hline
\end{tabular}


Table 3

Treatment Effects for Stress and Burnout

\begin{tabular}{lcccccr}
\hline & $\begin{array}{c}b \\
\text { Estimate }\end{array}$ & $\begin{array}{c}\text { Standard } \\
\text { Error }\end{array}$ & $\begin{array}{c}\text { T- } \\
\text { Statistic }\end{array}$ & DF & $p$ value \\
\hline Perceived Stress Scale & -0.53 & 0.22 & -2.36 & 23 & .03 & $*$ \\
Maslach Burnout & & & & & \\
Inventory & & & & & \\
Emotional Exhaustion & -0.30 & 0.21 & -1.43 & 23 & .17 \\
Depersonalization & -0.27 & 0.19 & -1.40 & 23 & .17 \\
Personal Accomplishment & 0.03 & 0.17 & 0.19 & 23 & .85 \\
Teacher Stress Scale & -0.08 & 0.20 & -0.41 & 23 & .69 \\
\hline
\end{tabular}

Notes. ${ }^{1}$ Each line is a separate 2 level multilevel model with Level 1-teacher, Level 2School, and schools were included as fixed effects. All models also controlled for the baseline measure, and years of teaching experience ${ }^{2}$ All measures were standardized. ${ }^{3}$ All $* p$ values are statistically significant using the Benjamini Hochberg method ${ }^{4} n=52$ teachers

Table 4

Treatment Effects for Coping Strategies

\begin{tabular}{|c|c|c|c|c|c|c|}
\hline & $\begin{array}{c}b \\
\text { Estimate }\end{array}$ & $\begin{array}{l}\text { Standard } \\
\text { Error }\end{array}$ & $\begin{array}{c}\mathrm{T}- \\
\text { Statistic }\end{array}$ & DF & $\begin{array}{c}p \\
\text { value }\end{array}$ & \\
\hline Acceptance & 0.13 & 0.22 & 0.57 & 23 & .57 & \\
\hline Active Coping & 0.55 & 0.24 & 2.32 & 23 & .03 & \\
\hline Humor & -0.23 & 0.24 & -0.96 & 23 & .35 & \\
\hline Instrumental Support & 0.22 & 0.24 & 0.91 & 23 & .37 & \\
\hline Planning & 0.62 & 0.23 & 2.73 & 23 & .01 & $*$ \\
\hline Positive Reframing & 0.66 & 0.24 & 2.74 & 23 & .01 & $*$ \\
\hline Religion & 0.01 & 0.18 & 0.03 & 23 & .98 & \\
\hline Use of Emotional Support & -0.04 & 0.24 & -0.17 & 23 & .87 & \\
\hline Self-Blame & -0.49 & 0.25 & -1.94 & 23 & .06 & \\
\hline Self-Distraction & -0.30 & 0.27 & -1.14 & 23 & .27 & \\
\hline Substance Use & -0.44 & 0.16 & -2.72 & 23 & .01 & $*$ \\
\hline Venting & -0.15 & 0.27 & -0.55 & 23 & .58 & \\
\hline
\end{tabular}

Notes. ${ }^{1}$ Each line is a separate 2 level multilevel model with Level 1-teacher, Level 2School, and schools were included as fixed effects. All models also controlled for the baseline measure, and years of teaching experience ${ }^{2}$ All measures were standardized. ${ }^{3}$ All * $p$ values are statistically significant using the Benjamini Hochberg method ${ }^{4} n=52$ teachers 
Table 5

Treatment Effects for Efficacy, Organizational Health, and Satisfaction

\begin{tabular}{lccccc}
\hline & $b$ & Standard & T- & DF & $p$ \\
Estimate & Error & Statistic & & value \\
\hline Teaching Efficacy & -0.12 & 0.20 & -0.63 & 23 & .54 \\
OHI - Collegial Leadership & 0.00 & 0.19 & 0.00 & 23 & .99 \\
OHI - Teacher Affiliation & -0.17 & 0.21 & -0.79 & 23 & .44 \\
Teacher Satisfaction & 0.23 & 0.15 & 1.49 & 23 & .15 \\
\hline
\end{tabular}

Notes. ${ }^{1}$ Each line is a separate 2 level multilevel model with Level 1-teacher, Level 2School, and schools were included as fixed effects. All models also controlled for the baseline measure, and years of teaching experience ${ }^{2}$ All measures were standardized. ${ }^{3}$ All $* p$ values are statistically significant using the Benjamini Hochberg method ${ }^{4} n=52$ teachers

Table 6

Treatment Effects for Mental Health Screening

\begin{tabular}{lcccccc} 
& $b$ & Standard & T- & DF & \multicolumn{2}{c}{} \\
& Estimate & Error & Statistic & & value & \\
\hline GAD -7 & -0.51 & 0.19 & -2.64 & 23 & .01 & $*$ \\
PHQ -8 & -0.40 & 0.22 & -1.82 & 23 & .08 & \\
\hline
\end{tabular}

Notes. ${ }^{1}$ Each line is a separate 2 level multilevel model with Level 1-teacher, Level 2School, and schools were included as fixed effects. All models also controlled for the baseline measure, and years of teaching experience ${ }^{2}$ All measures were standardized. ${ }^{3}$ All $* p$ values are statistically significant using the Benjamini Hochberg method ${ }^{4} n=52$ teachers

Table 7

Treatment Effects for Observed Teacher Behaviors

\begin{tabular}{|c|c|c|c|c|c|}
\hline & $\begin{array}{c}b \\
\text { Estimate }\end{array}$ & $\begin{array}{c}\text { Standard } \\
\text { Error }\end{array}$ & $\begin{array}{c}\mathrm{T}- \\
\text { Statistic }\end{array}$ & DF & $\begin{array}{c}p \\
\text { value }\end{array}$ \\
\hline BCIO - Negative Implementation ${ }^{4}$ & 0.03 & 0.21 & 0.13 & 22 & .89 \\
\hline BCIO - Positive Implementation ${ }^{4}$ & 0.15 & 0.29 & 0.50 & 22 & .62 \\
\hline Classroom Atmosphere Scale ${ }^{5}$ & 0.20 & 0.26 & 0.77 & 21 & .45 \\
\hline Overall Rating Scale S $^{4}$ & 0.25 & 0.27 & 0.91 & 22 & .38 \\
\hline
\end{tabular}

Notes. ${ }^{1}$ Each line is a separate 2 level multilevel model with Level 1-teacher, Level 2School, and schools were included as fixed effects. All models also controlled for the baseline measure, and years of teaching experience ${ }^{2}$ All measures were standardized. ${ }^{3}$ All $* p$ values are statistically significant using the Benjamini Hochberg method ${ }^{4} n=51,{ }^{5} n$ $=49$ 
Table 8

Growth Modelling of Single-Item Measures of Stress and Coping over Time

\begin{tabular}{|c|c|c|c|c|c|c|}
\hline & $\begin{array}{c}b \\
\text { Estimate }\end{array}$ & $\begin{array}{l}\text { Standard } \\
\text { Error }\end{array}$ & $\begin{array}{c}\mathrm{T}- \\
\text { Statistic }\end{array}$ & $\mathrm{DF}$ & $\begin{array}{c}p \\
\text { value }\end{array}$ & \\
\hline \multicolumn{7}{|l|}{ Stress } \\
\hline Intercept & 6.52 & 0.45 & 14.33 & 208 & $<.001$ & $*$ \\
\hline Time & -0.01 & 0.12 & -0.06 & 208 & .95 & \\
\hline Intervention & 0.75 & 0.63 & 1.19 & 25 & .24 & \\
\hline Time:Intervention & -0.22 & 0.17 & -1.25 & 208 & .21 & \\
\hline \multicolumn{7}{|l|}{ Coping } \\
\hline Intercept & 5.88 & 0.42 & 13.83 & 208 & $<.001$ & $*$ \\
\hline Time & 0.15 & 0.11 & 1.35 & 208 & .18 & \\
\hline Intervention & -0.53 & 0.60 & -0.89 & 25 & .38 & \\
\hline Time:Intervention & 0.38 & 0.15 & 2.57 & 208 & .01 & $*$ \\
\hline \multicolumn{7}{|l|}{ Confidence in Coping } \\
\hline Intercept & 6.57 & 0.42 & 15.50 & 208 & $<.001$ & $*$ \\
\hline Time & -0.01 & 0.08 & -0.15 & 208 & .88 & \\
\hline Intervention & -0.67 & 0.59 & -1.13 & 25 & .27 & \\
\hline Time:Intervention & 0.38 & 0.12 & 3.23 & 208 & .001 & $*$ \\
\hline \multicolumn{7}{|c|}{ Awareness of Coping Strategies } \\
\hline Intercept & 5.74 & 0.47 & 12.17 & 208 & $<.001$ & $*$ \\
\hline Time & 0.36 & 0.12 & 3.13 & 208 & .002 & $*$ \\
\hline Intervention & 0.78 & 0.66 & 1.16 & 25 & .26 & \\
\hline Time:Intervention & 0.18 & 0.16 & 1.14 & 208 & .26 & \\
\hline \multicolumn{7}{|l|}{ Understanding of Coping } \\
\hline \multicolumn{7}{|l|}{ Strategies } \\
\hline Intercept & 5.06 & 0.46 & 10.87 & 208 & $<.001$ & $*$ \\
\hline Time & 0.44 & 0.11 & 4.03 & 208 & $<.001$ & $*$ \\
\hline Intervention & 0.10 & 0.64 & 0.15 & 25 & .88 & \\
\hline Time:Intervention & 0.22 & 0.15 & 1.51 & 208 & .13 & \\
\hline \multicolumn{7}{|l|}{ Use of Coping Strategies } \\
\hline Intercept & 4.70 & 0.47 & 9.94 & 208 & $<.001$ & $*$ \\
\hline Time & 0.32 & 0.10 & 3.24 & 208 & .001 & $*$ \\
\hline Intervention & 0.23 & 0.66 & 0.35 & 25 & .73 & \\
\hline Time:Intervention & 0.37 & 0.14 & 2.69 & 208 & .008 & $*$ \\
\hline
\end{tabular}

Notes. ${ }^{1}$ All multilevel growth models included 3 levels: Level 1 - Time, Level 2 -

Teacher, Level $3-$ School. ${ }^{2}$ All $* p$ values are statistically significant using the Benjamini Hochberg method ${ }^{3} n=50$ teachers, 2 teachers did not complete all of the weekly rating forms and were removed with listwise deletion 
Table 9

Pre-Post Comparisons of Study Measures $(N=52)$

\begin{tabular}{|c|c|c|c|c|c|c|c|c|c|}
\hline & \multicolumn{4}{|c|}{ Control } & \multicolumn{4}{|c|}{ Treatment } & \multirow[b]{3}{*}{ Effect } \\
\hline & \multicolumn{2}{|c|}{ Pre } & \multicolumn{2}{|c|}{ Post } & \multicolumn{2}{|c|}{ Pre } & \multicolumn{2}{|c|}{ Post } & \\
\hline & Mean & $\underline{(\mathrm{SD})}$ & Mean & $\underline{(\mathrm{SD})}$ & Mean & $\underline{(\mathrm{SD})}$ & Mean & $\underline{(\mathrm{SD})}$ & \\
\hline \multicolumn{10}{|l|}{ Stress and Burnout } \\
\hline Perceived Stress Scale & 19.77 & 5.23 & 19.27 & 5.55 & 21.62 & 6.20 & 17.38 & 6.16 & -0.64 \\
\hline Emotional Exhaustion & 38.12 & 11.10 & 39.65 & 11.01 & 41.08 & 12.42 & 37.96 & 12.10 & -0.40 \\
\hline Depersonalization & 14.08 & 6.69 & 14.19 & 6.67 & 13.31 & 5.92 & 12.69 & 5.24 & -0.12 \\
\hline Personal Accomplishment & 44.81 & 7.27 & 44.73 & 7.48 & 46.54 & 3.42 & 46.58 & 6.05 & 0.02 \\
\hline Teacher Stress Scale & 60.58 & 10.08 & 60.92 & 11.29 & 61.54 & 11.22 & 59.54 & 10.46 & -0.22 \\
\hline \multicolumn{10}{|l|}{ Single-Item Measures } \\
\hline Stress & 7.85 & 1.29 & 7.54 & 1.68 & 8.04 & 1.68 & 7.50 & 2.27 & -0.13 \\
\hline Coping & 5.42 & 2.30 & 6.35 & 1.98 & 5.27 & 1.93 & 7.38 & 2.02 & 0.57 \\
\hline Confidence in Coping & 6.54 & 2.25 & 6.62 & 2.06 & 6.38 & 2.06 & 7.54 & 1.86 & 0.52 \\
\hline Awareness & 5.46 & 2.79 & 7.04 & 1.95 & 6.69 & 2.38 & 9.00 & 1.02 & 0.34 \\
\hline Use of Coping & 4.46 & 2.86 & 6.08 & 2.58 & 5.23 & 2.30 & 8.15 & 1.41 & 0.55 \\
\hline Understanding & 5.15 & 2.20 & 6.92 & 2.13 & 5.46 & 2.61 & 8.15 & 1.99 & 0.41 \\
\hline \multicolumn{10}{|l|}{ Brief Cope } \\
\hline Active Coping & 5.85 & 1.57 & 5.88 & 1.40 & 5.77 & 1.34 & 6.38 & 1.39 & 0.41 \\
\hline Instrumental Support & 5.73 & 1.56 & 5.54 & 1.27 & 5.58 & 1.92 & 5.88 & 1.51 & 0.31 \\
\hline Planning & 6.04 & 1.51 & 5.88 & 1.11 & 5.85 & 1.46 & 6.58 & 1.27 & 0.66 \\
\hline Positive Reframing & 6.15 & 1.52 & 5.50 & 1.66 & 6.12 & 1.48 & 6.46 & 1.17 & 0.67 \\
\hline Use of Emotional Support & 5.73 & 1.48 & 5.81 & 1.41 & 5.31 & 1.93 & 5.69 & 1.81 & 0.18 \\
\hline Self-Blame & 5.35 & 1.70 & 5.12 & 1.93 & 5.27 & 1.69 & 4.12 & 1.80 & -0.52 \\
\hline Self-Distraction & 5.85 & 1.46 & 5.77 & 1.34 & 5.81 & 1.67 & 5.19 & 1.67 & -0.35 \\
\hline Substance Use & 1.58 & 0.86 & 1.81 & 1.06 & 1.35 & 0.63 & 1.31 & 0.55 & -0.34 \\
\hline Venting & 4.85 & 1.52 & 5.23 & 1.37 & 5.04 & 1.46 & 5.12 & 1.61 & -0.20 \\
\hline \multicolumn{10}{|l|}{ Mental Health } \\
\hline $\mathrm{GAD}-7$ & 9.54 & 4.69 & 10.19 & 5.02 & 12.08 & 5.77 & 9.15 & 5.69 & -0.67 \\
\hline PHQ - 8 & 9.19 & 5.61 & 9.38 & 5.71 & 10.54 & 6.04 & 7.88 & 6.40 & -0.48 \\
\hline \multicolumn{10}{|l|}{ Contextual Factors } \\
\hline Teaching Efficacy & 77.58 & 11.88 & 82.58 & 13.54 & 79.92 & 9.64 & 83.69 & 13.75 & -0.10 \\
\hline OHI - Collegial Leadership & 30.81 & 8.10 & 29.50 & 8.24 & 30.92 & 7.22 & 30.81 & 8.10 & 0.15 \\
\hline OHI - Teacher Affiliation & 26.19 & 4.68 & 26.31 & 4.84 & 25.96 & 4.89 & 25.42 & 4.22 & -0.14 \\
\hline Teacher Satisfaction & 14.62 & 3.16 & 14.42 & 3.47 & 14.35 & 3.15 & 14.88 & 3.22 & 0.22 \\
\hline
\end{tabular}


Figure 1. Average Self-Reported Stress Over Time $(\mathrm{N}=50)$

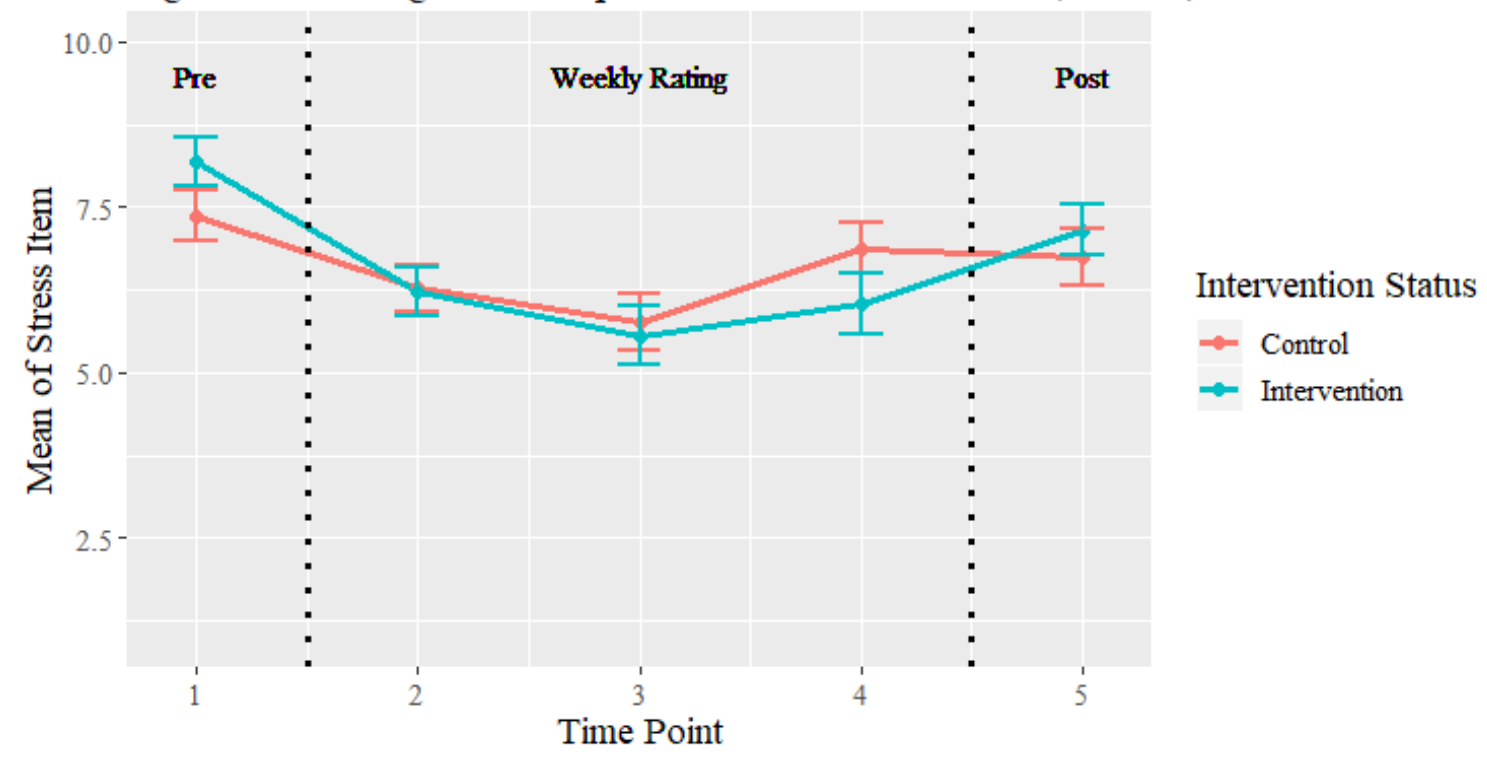

Figure 2. Average Self-Reported Coping Over Time $(\mathrm{N}=50)$

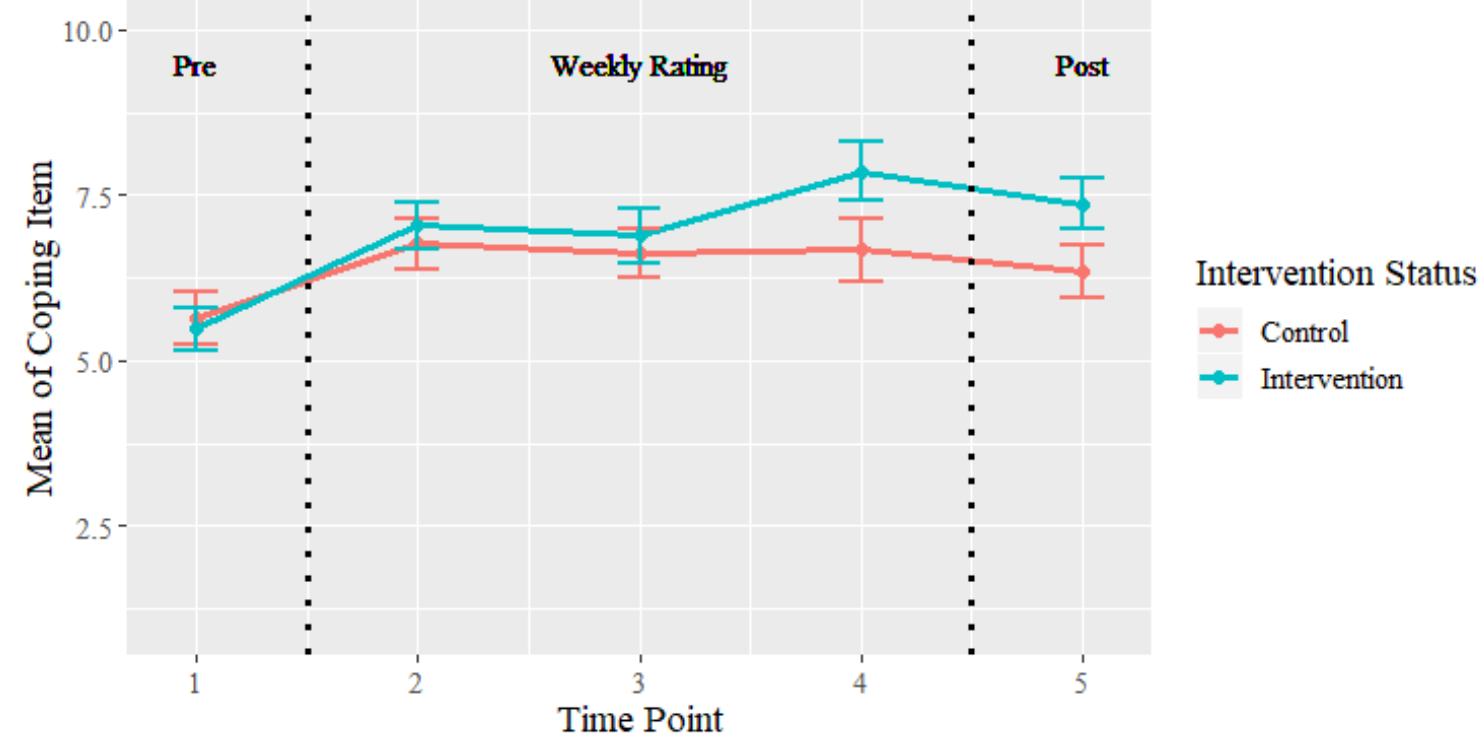

Notes. ${ }^{1}$ Error bars represent the standard error of the mean. ${ }^{2}$ Dotted lines represent the timing of the beginning the intervention and the end of the intervention. ${ }^{3}$ Post intervention follow up was completed 4-6 weeks after the intervention began. 
Figure 3. Average Self-Reported Confidence in Coping Over Time $(\mathrm{N}=50)$

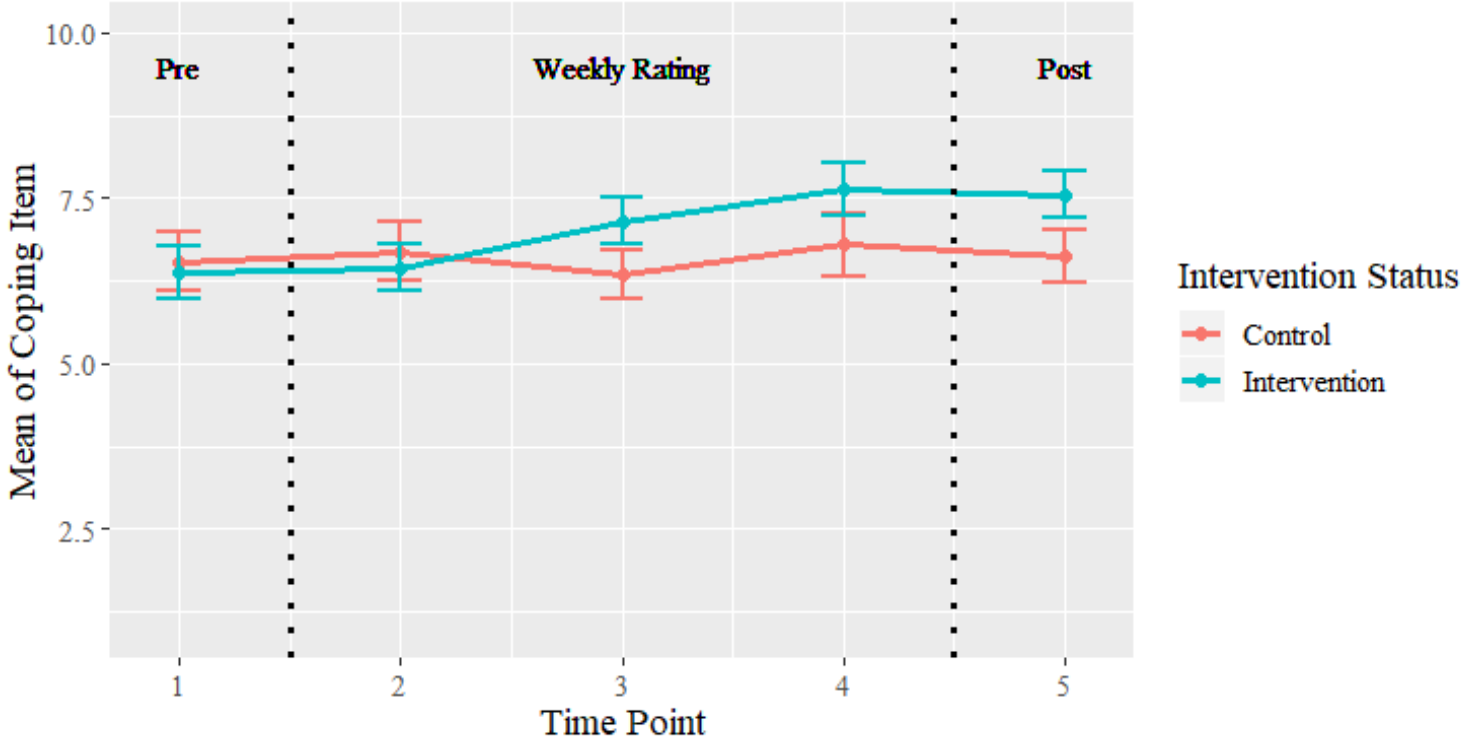

Figure 4. Average Self-Reported Awareness of Coping Strategies Over Time

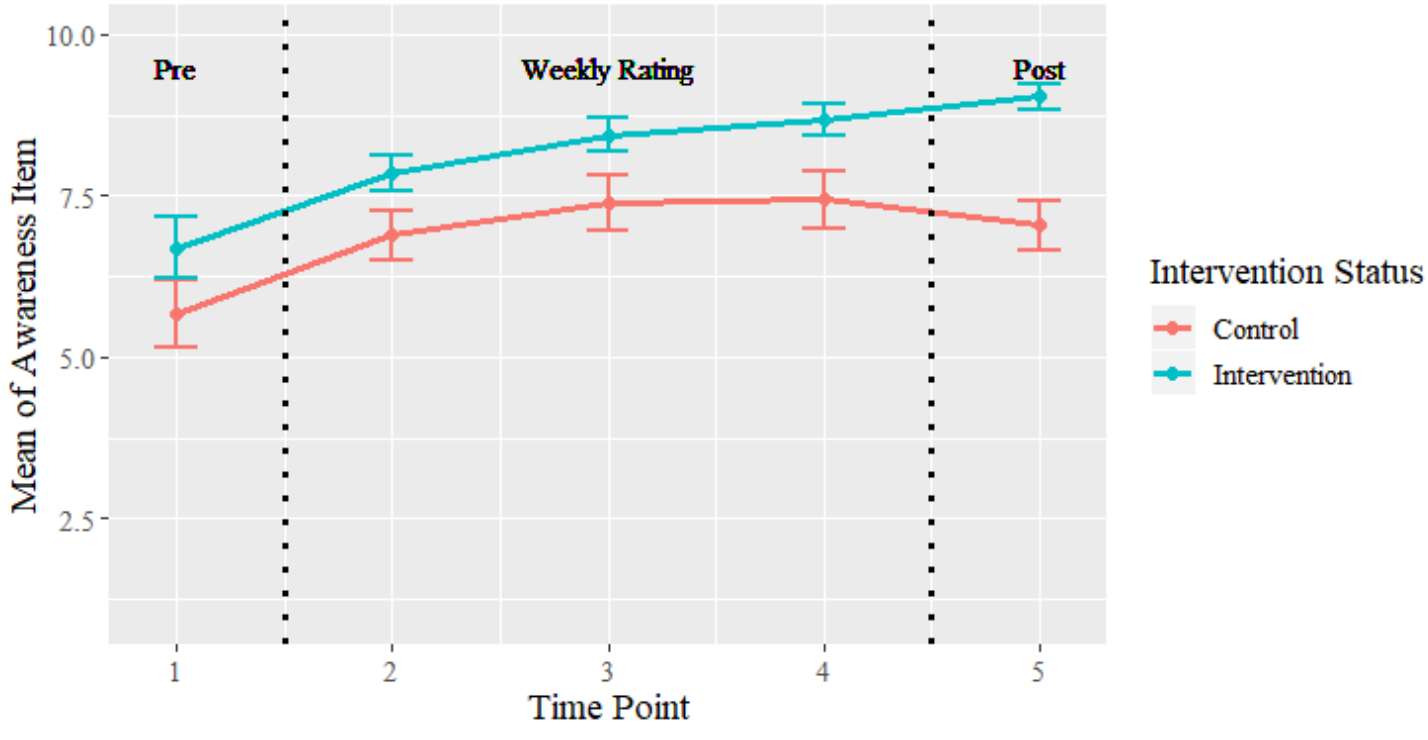


Figure 5. Self-Reported Understanding of Coping Over Time $(\mathrm{N}=50)$

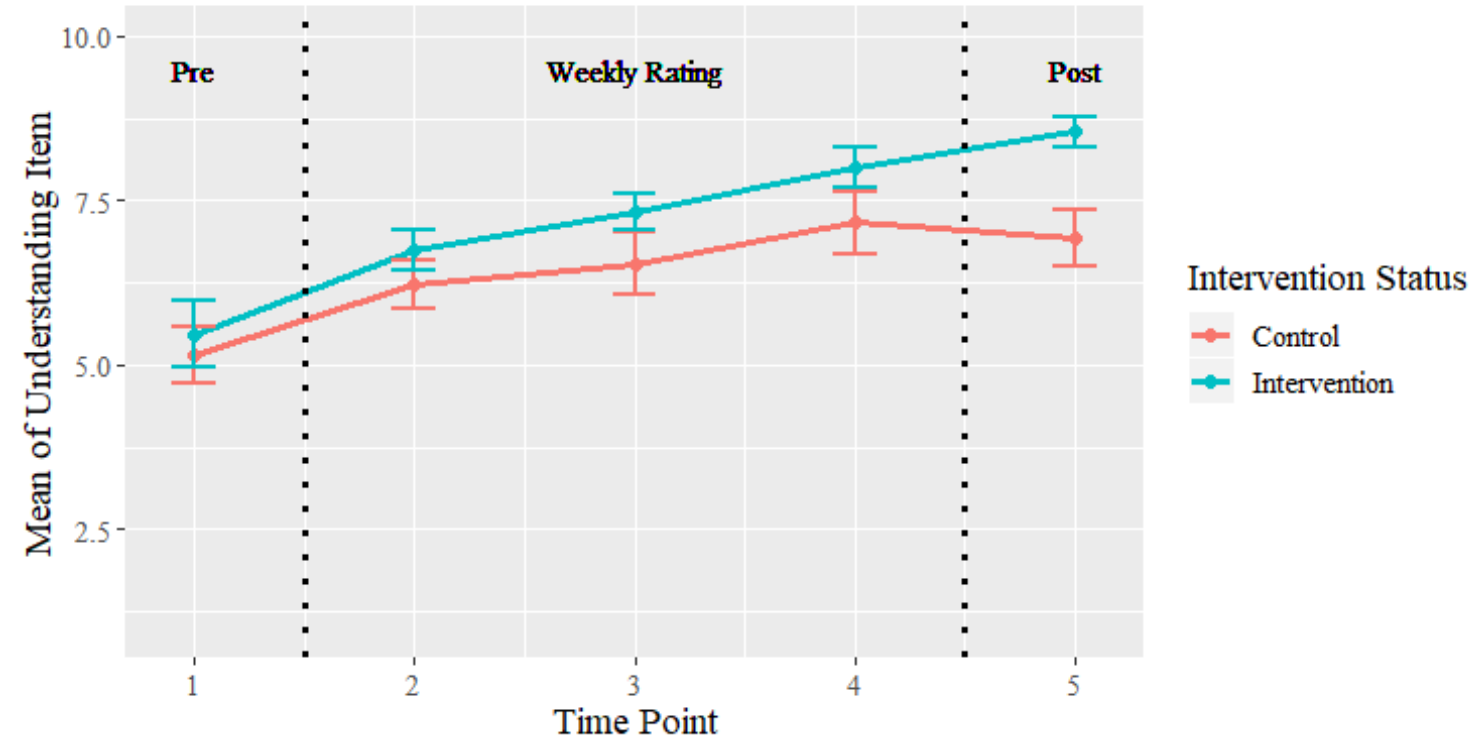

Figure 6. Average Self-Reported Use of Coping Over Time $(\mathrm{N}=50)$

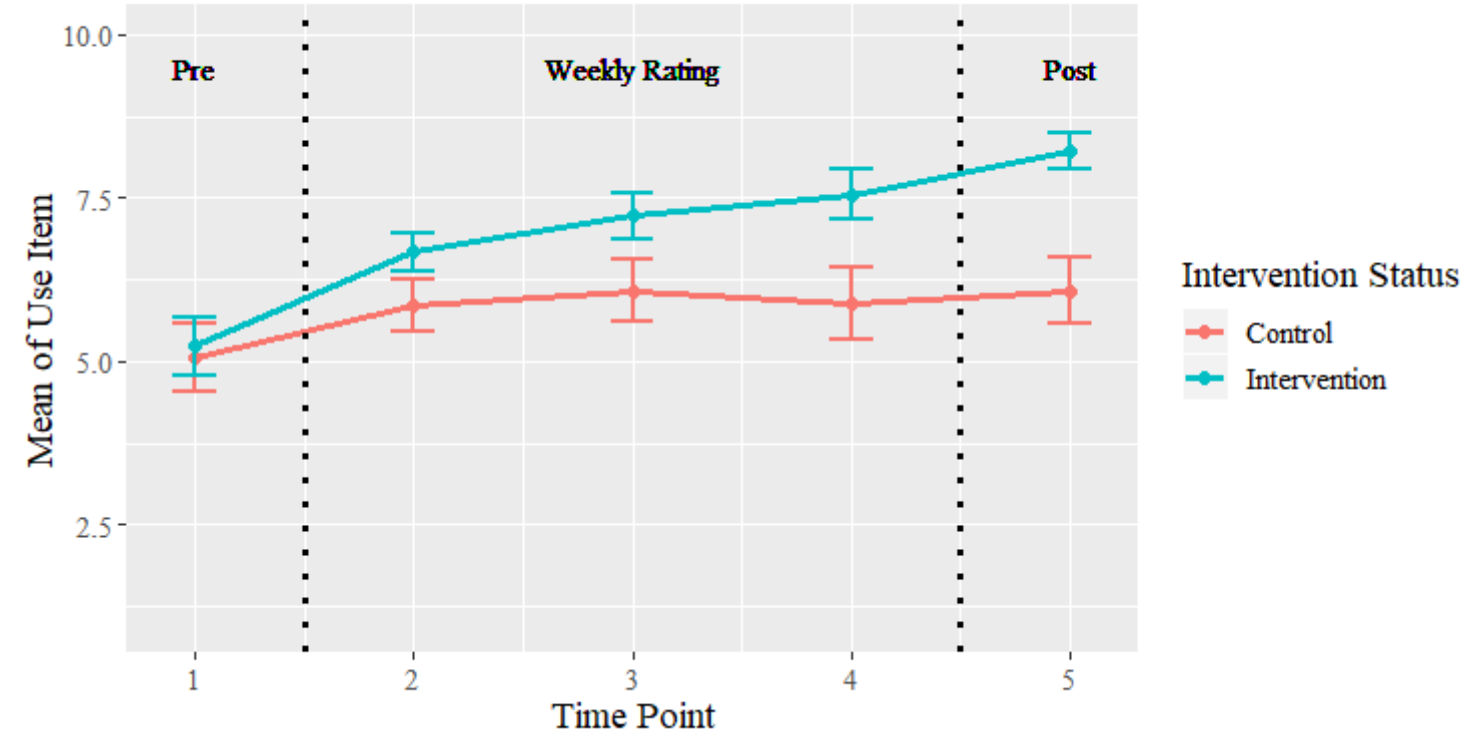


Figure 7. Participants' change in anxiety severity level within treatment and control groups $(\mathrm{N}=52)$

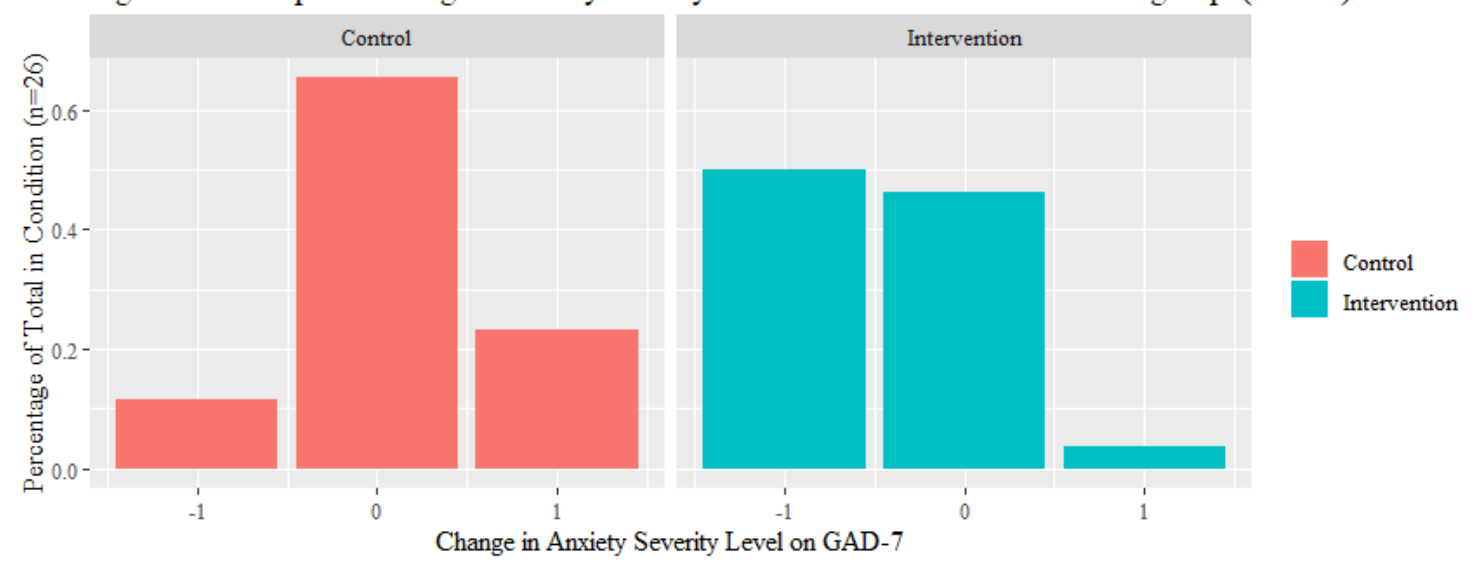

Notes. The change in anxiety severity level (none, mild, moderate, severe) was calculated by examining the change from pre to post on the GAD-7. The graph represents the percentage from each group who increased (1), stayed the same (0), or decreased (-1) in symptom severity levels.

Figure 8. Participants' change in depression severity level within treatment and control groups $(\mathrm{N}=52)$

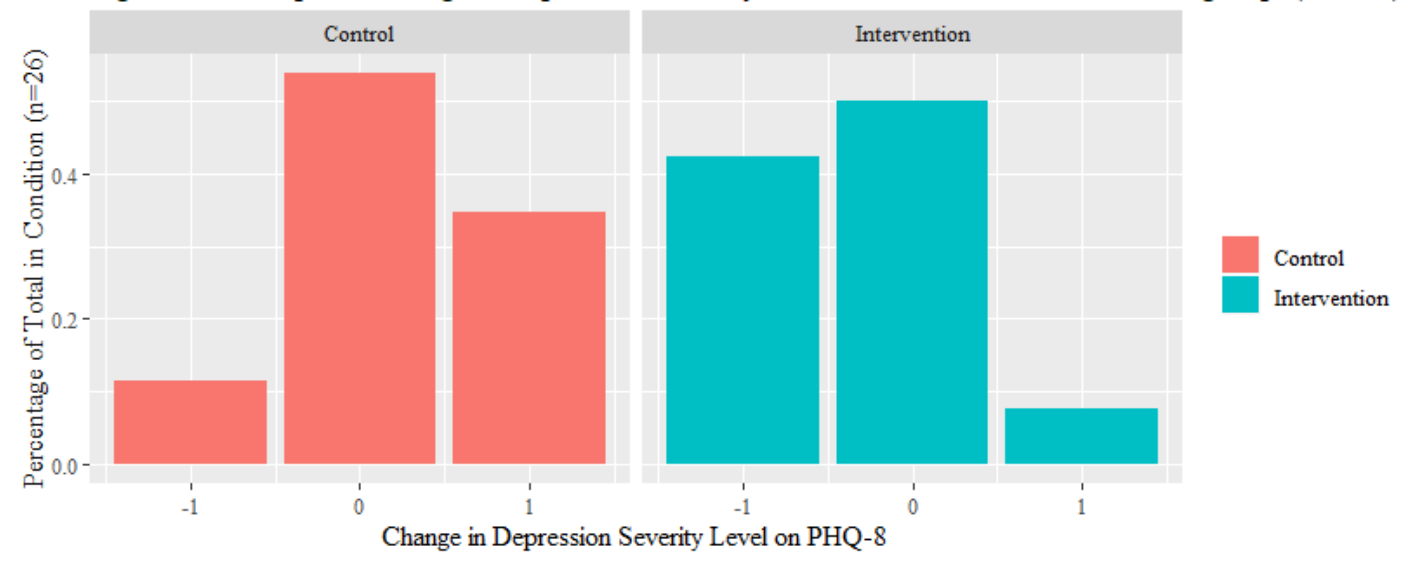

Notes. The change in depression severity level (none, mild, moderate, severe) was calculated by examining the change from pre to post on the PHQ-8. The graph represents the percentage from each group who increased (1), stayed the same (0), or decreased (-1) in symptom severity levels. 
Table 10

Control Group Only Pre-Post Comparisons $(n=19)$

\begin{tabular}{|c|c|c|c|c|c|c|c|}
\hline & \multicolumn{2}{|c|}{$\begin{array}{c}\text { Pre } \\
\text { Intervention } \\
\text { Time } 1 \\
\end{array}$} & \multicolumn{2}{|c|}{$\begin{array}{c}\text { Pre } \\
\text { Intervention } \\
\text { Time } 2 \\
\end{array}$} & \multicolumn{2}{|c|}{$\begin{array}{c}\text { Post } \\
\text { Intervention } \\
\text { Time } 3 \\
\end{array}$} & \multirow[t]{2}{*}{$\begin{array}{c}\text { Cohen's } \\
\text { D } \\
\text { T2 T3 }\end{array}$} \\
\hline & Mean & $\mathrm{SD}$ & Mean & $\mathrm{SD}$ & Mean & SD & \\
\hline $\begin{array}{l}\text { Perceived Stress Scale } \\
\text { Maslach Burnout } \\
\text { Inventory }\end{array}$ & 19.81 & 5.13 & 19.44 & 5.52 & 14.95 & 4.89 & -0.78 \\
\hline Emotional Exhaustion & 38.22 & 10.9 & 40.15 & 11.1 & 38.11 & -10.75 & -0.17 \\
\hline $\begin{array}{l}\text { Depersonalization } \\
\text { Personal }\end{array}$ & 14.04 & 6.56 & 14.3 & 6.56 & 15.42 & 5.76 & 0.16 \\
\hline Accomplishment & 44.85 & 7.13 & 44.96 & 7.43 & 46.21 & 6.42 & 0.16 \\
\hline Stress & 7.85 & 1.26 & 7.56 & 1.65 & 7.16 & 1.86 & -0.21 \\
\hline Coping & 5.48 & 2.28 & 6.37 & 1.94 & 7.32 & 1.95 & $0.44 *$ \\
\hline Confidence in Coping & 6.56 & 2.21 & 6.63 & 2.02 & 7.74 & 1.94 & 0.51 \\
\hline $\begin{array}{l}\text { Awareness of Coping } \\
\text { Understanding of }\end{array}$ & 5.52 & 2.75 & 7.11 & 1.95 & 9.21 & 1.08 & $1.20 *$ \\
\hline Coping & 5.26 & 2.23 & 6.96 & 2.10 & 8.58 & 1.84 & $0.74 *$ \\
\hline Use of Coping & 4.52 & 2.82 & 6.15 & 2.55 & 7.63 & 2.11 & $0.57 *$ \\
\hline Active Coping & 5.85 & 1.54 & 5.89 & 1.37 & 6.15 & 1.46 & 0.17 \\
\hline Instrumental Support & 5.81 & 1.59 & 5.63 & 1.33 & 5.35 & 1.42 & -0.18 \\
\hline Planning & 6.04 & 1.48 & 5.89 & 1.09 & 6.2 & 1.28 & 0.24 \\
\hline $\begin{array}{l}\text { Positive Reframing } \\
\text { Use of Emotional }\end{array}$ & 6.15 & 1.49 & 5.52 & 1.63 & 6.45 & 1.28 & 0.57 \\
\hline Support & 5.78 & 1.48 & 5.85 & 1.41 & 5.65 & 1.57 & -0.12 \\
\hline Self-Blame & 5.3 & 1.68 & 5.11 & 1.89 & 4.3 & 1.78 & -0.40 \\
\hline Self-Distraction & 5.93 & 1.49 & 5.74 & 1.32 & 5.15 & 1.53 & -0.37 \\
\hline Substance Use & 1.59 & 0.84 & 1.81 & 1.04 & 1.3 & 0.66 & -0.53 \\
\hline Venting & 4.89 & 1.5 & 5.3 & 1.38 & 5.1 & 1.45 & -0.13 \\
\hline $\mathrm{GAD}-7$ & 9.81 & 4.82 & 10.59 & 5.34 & 7.95 & 5.24 & -0.45 \\
\hline PHQ - 8 & 9.00 & 5.59 & 9.56 & 5.67 & 7.37 & 6.47 & -0.33 \\
\hline
\end{tabular}

Notes. * indicates statistically significant with Benjamini Hochberg correction 


\section{Appendix F.}

\section{Feedback on the Program.}

What did you like best about the Stress Management for Teachers program overall?

- The program was easy to follow and full of helpful information.

- I like the opportunity to self-evaluate how stress effects me as a teacher. I enjoyed the strategies and information.

- It was nice to know that I wasn't alone and everyone else was feeling the same stress and pressure.

- New ideas to handle stress. Kindness and professionalism of trainers and observers

- in-person training and the book

- It wasn't hard and it didn't feel like it was another item on my to do list.

- It made me far more aware of the stress I have, my ability to cope with it and when I realized I wasn't coping well, it reminded me to make a change the following week.

- The in person meeting.

- A new outlook on stress in my life!

- Meeting with Colleen and collaborating with my peer to discuss strategies.

- I love that I felt like I had someone to support me. It didn't feel like I was in this alone.

- The resources I got and strategies I learned

- Help with stress

- The in-person training was great!

- I do not feel I can pick the best, truly, I thought the program as a WHOLE was beneficial.

- The management tips for controlling stress.

- Getting paid

- Collaboration with other teachers.

- I mostly enjoyed the provision of resources and implementation instructions.

- It made me much more aware of the impacts that stress can have on my longterm health and emphasized to me that it is of upmost priority to get the stress under control. For me, the stress is not only just about the job, but more about how I react to all things in my life. The course allowed me to see that.

- I feel like, even when I was a bit overwhelmed, it was a good provider of one small change at a time that I thought I could handle to improve my stress levels.

- I like that there is an effort to help teachers and support their mental health.

- I actually learned a few new techniques that I had not tried in the past.

- Great information, procedures and presentation. 
- The fact that there are a variety of options available, especially if individuals do not have any ideas about how to handle stress due to work and/or personal life.

- It was nice to be accountable for my emotions weekly.

- That most of it was online

- To make me more mindful of my mental health

- It was very beneficial to be heard about what stressors teachers experience and to have my stress and pressure validated and to learn about strategies to deal with stress and limit stress.

- It helps teachers

- I liked how laid back it was because we do not need more stress added to our lives!

- Nice to know about more strategies.

- Acknowledging the positive parts of our lives, even when there is stress. Keep your eyes up.

- meeting others

- Receiving in oerson training and the book.

- the usefulness of the content and the sensitivity of the staff

- That it was only JC School District teachers. Set up in a community.

- Making me aware of my stress and sort of forcing me to evaluate myself regularly.

- The goal.

- Participating in this program made me more aware that I can do something about stress in my life.

- How easy it was for me to do throughout my day.

- The strategies were very helpful. There were many I did not know about and tried.

- Surveys-they made me evaluate myself/stress level.

- This is helpful because mental health is such an overlooked area for teachers.

- I learned a lot and tried a lot of new coping skills!

\section{What did you like least about the Stress Management for Teachers program} overall?

- The time seemed to go so quickly. The information is extremely helpful, but hard to get through all that is needed and finish all other work as a first year teacher.

- It is hard to fit in one more thing during the school year.

- nothing

- length of in person training

- online components

- The observations did not last that long so I am not sure how "good" my observation was.

- Nothing. I often forgot survey's and needed reminding but as never bothered by having to take them. 


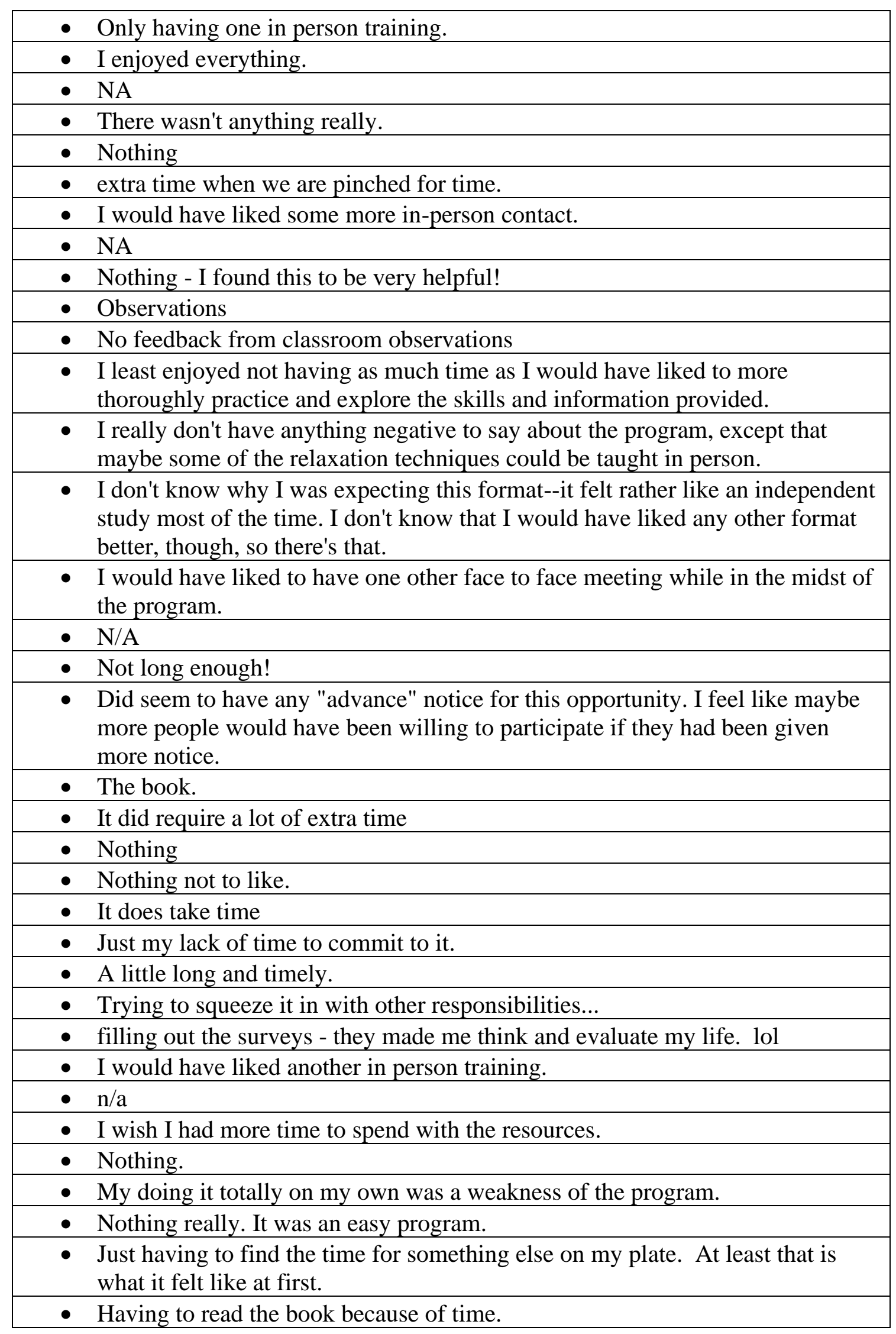


- More opportunities for daily application.

- I mean it is adding one more thing to do in a week, but that was my choice.

\section{VITA}

Colleen L. Eddy is a $5^{\text {th }}$ year doctoral candidate in Counseling Psychology at the University of Missouri. She has been a graduate research assistant with the Missouri Prevention Science Institute for nearly 5 years. Her clinical work focuses on youth mental health and she has had practicum placements providing evidence-based treatment in outpatient, home, and school settings. She will begin a pre-doctoral internship with Devereux Advanced Behavioral Health and the Children's Behavioral Health Services in Villanova, PA from August 2020 to August 2021. 